\title{
Mesoscale cyclic crystal plasticity with dislocation substructures
}

\author{
Gustavo M. Castelluccio ${ }^{1}$ and David L. McDowell ${ }^{1,3}$ \\ ${ }^{1}$ School of Aerospace, Transport and Manufacturing, Cranfield University, Bedfordshire, MK43 0AL, \\ UK. \\ ${ }^{2,3}$ Woodruff School of Mechanical Engineering and School of Materials Science and Engineering, \\ Georgia Institute of Technology, Atlanta, GA, USA
}

\section{Abstract}

Constitutive formulations have increasingly focused on physically-based approaches that are less phenomenological and incorporate information from multiple scales. Most dislocation-based plasticity approaches reflect many-body dislocation physics without considering the length scales introduced by the self-organization of dislocations into mesoscale structures. These structures promote internal stresses or back stresses that are heterogeneous and long-range in nature and play a critical intermediary role in distinguishing the stress at micro- and nano-scales under cyclic loading. We present a framework that explicitly incorporates length-scales and evolution laws associated with mesoscale dislocation substructures such as cells and persistent slip bands (PSBs) in metallic materials under cyclic loading. A physically-based formulation for the back stress based on the Eshelby inclusion formalism is introduced that explicitly depends on morphology of mesoscale dislocation structures. The approach employs material parameters that can be measured or computed at lower length scales to contrast the response of models and experiments for multiple single crystals orientation and polycrystals for a wide range of strains.

\section{Introduction}

Traditionally, constitutive models have attempted to match experimental behavior with largely ad-hoc mathematical formulations with adjustable material parameters. These parameters have typically been selected to match simulations to experiments at the macroscale by minimizing likelihood functions, e.g., fitting parameters with a least square error methodology. This approach is particularly problematic for models with parameters that are not uniquely defined by least squares error minimization, i.e., equally good matching to experiments can be achieved by multiple parameter values and is sensitive to the initial values assigned in the search process. The number of fitting parameters can be reduced by employing physicallybased constitutive model forms for which parameters can be either measured experimentally or computed using more predictive and small scale discrete models, e.g., density functional theory, molecular dynamics, and dislocation dynamics. Even when these parameters may carry large uncertainties, they can usually be bounded, which helps quantifying the underlying total variability of the constitutive model independently from macroscopic experiments.

Some efforts have emphasized the use of constitutive models that reduce the extent of parameter fitting to macroscopic experimental data. For instance, Kubin and coworkers (Kubin et al., 2008) proposed a model for pure face centered cubic (FCC) metals under monotonic loading that avoids empiricism in parameter fitting by computing dislocation interaction parameters via dislocation dynamics. However, such approaches become significantly more complex for more highly alloyed systems or non-monotonic loading. Sauzay and coworkers (Sauzay et al., 2014; Steckmeyer et al., 2012) developed crystal plasticity constitutive models to predict the response FCC single crystal under cyclic conditions, and they limited the number of fitting constants to a few. These constants primarily affect the back stress due to a lack of information from bottom-up approaches or experimental measurements.

1 | P a g e

Published by Elsevier. This is the Author Accepted Manuscript issued with:

Creative Commons Attribution Non-Commercial No Derivatives License (CC:BY:NC:ND 4.0).

The final published version (version of record) is available online at DOI:10.1016/j.ijplas.2017.06.002.

Please refer to any applicable publisher terms of use. 
This paper simulates the cyclic response of FCC single- and polycrystals with a constitutive model with parameters that can be measured experimentally or quantified using bottom-up approaches such as atomistic modeling and dislocation dynamics. We propose physically-based models for each dominant deformation mechanism while the uncertainty of model parameters can be estimated independent of the measured macroscopic response. The bounds of parameters provide a means to assess confidence in the proposed mechanisms and inform decisions to discard models that do not match the expected macroscopic response. Accordingly, this approach facilitates model verification and validation at multiple scales.

\section{Fundamentals of physically-based constitutive modeling}

We consider physically-based crystal plasticity formulations that describe observed dislocation mechanisms for medium to high stacking fault energy FCC metals. Our goal is to match experimental data by avoiding the introduction of "fitting" parameters without a clear physical basis. This means that all parameters should be bounded based on experimental data or lower scale simulation results. In this regard, we distinguish between material parameters that can be measured directly with low uncertainty (e.g., Boltzmann constant, elastic stiffness) and parameters that represent stochastic processes of much higher uncertainty (e.g., mean strength of dislocation-dislocation interactions and the characteristic evolving dislocation mean free path).

\section{Atomistic scale: Dislocation energy barriers}

The flow rule in crystal plasticity as proposed by Asaro (R. J. Asaro, 1983; Robert J. Asaro, 1983; Asaro and Needleman, 1985) specifies the shearing rate for each of the 12 slip systems in FCC crystals. Kocks et al. (Kocks et al., 1975) proposed to quantify the slip system shearing rate for the $\alpha^{\text {th }}$ slip system in the thermally activated flow regime of dislocation bypass of barriers according to

$$
\dot{\gamma}^{\alpha}=\dot{\gamma}_{o}^{\alpha} \exp \left(-\frac{\Delta G\left(\tau_{e f f}^{\alpha}\right)}{k_{B} T}\right) \operatorname{sgn}\left(\tau^{\alpha}-B^{\alpha}\right)
$$

Here, $\dot{\gamma}_{o}^{\alpha}$ is the reference shearing rate, $\Delta G$ is the energy barrier for obstacle bypass which depends on the effective stress $\tau_{\text {eff }}^{\alpha}, k_{B}$ it the Boltzmann constant, $B^{\alpha}$ is the directional intragranular slip system back stress, and $T$ is the absolute temperature in $\mathrm{K}$.

Equation (1) corresponds to an Arrhenius temperature dependence on the energy barrier that a dislocation must overcome via thermal activation to result in irreversible plastic deformation. The effective shear stress $\left(\tau_{\text {eff }}^{\alpha}\right)$ corresponds to the local stress that drives dislocation glide. The effective stress differs from the macroscopic resolved stress due to the stress shielding effects of mesoscopic dislocation structures such as dislocation cells and walls; this distinction holds for both single- and polycrystals. The activation enthalpy $\Delta G$ pertains to one type of barrier or the mean effective barrier, and has been parameterized by Kocks et al. (Kocks et al., 1975; Kocks and Mecking, 2003) in the form

$$
\Delta G\left(\tau_{\text {eff }}^{\alpha}\right)=F_{0}\left(1-\left[\frac{\tau_{e f f}^{\alpha}}{s_{t}^{0} \frac{\mu}{\mu_{0}}}\right]^{p}\right)^{q}
$$


Here, $F_{0}$ and $p, q$ are the activation energy and profile parameters, respectively, for dislocation barrier bypass at $\tau_{\text {eff }}^{\alpha}=0, s_{t}^{0}$ is thermal slip resistance at $0 \mathrm{~K}$, and $\mu$ and $\mu_{0}$ represent the shear modulus $\left(\mathrm{C}_{44}\right)$ at temperature $T$ and $0 \mathrm{~K}$, respectively.

We assume that a single set of parameters can describe the dominant weakest link or the statistical ensemble of dislocations. The effective stress that drives the barrier bypass at the slip system level is given by

$$
\tau_{e f f}^{\alpha}=\left\langle\left|\tau^{\alpha}-B^{\alpha}\right|-S^{\alpha}\right\rangle
$$

where $\tau^{\alpha}$ is the local slip system resolved shear stress and $S^{\alpha}$ is the nondirectional threshold stress. In addition, $\langle g\rangle=0$ if $g \leq 0,\langle g\rangle=g$ if $g>0$.

\section{Mesoscale dislocation structures under cyclic loading}

Li et al. (Li et al., 2011) reviewed experimental work and highlighted similarities among dislocation substructures in FCC single crystals after being cycled along different orientations. They considered three pure metals with increasing stable stacking fault energies (SFEs): $\mathrm{Ag}\left(15 \mathrm{mJm}^{-2}\right), \mathrm{Cu}\left(40 \mathrm{mJm}^{-2}\right)$ and Ni $\left(130 \mathrm{mJm}^{-2}\right)$. Figure 1 summarizes the structures found after cyclic loading of single crystals at medium strain ranges (high cycle fatigue) as a function of the uniaxial loading orientation.

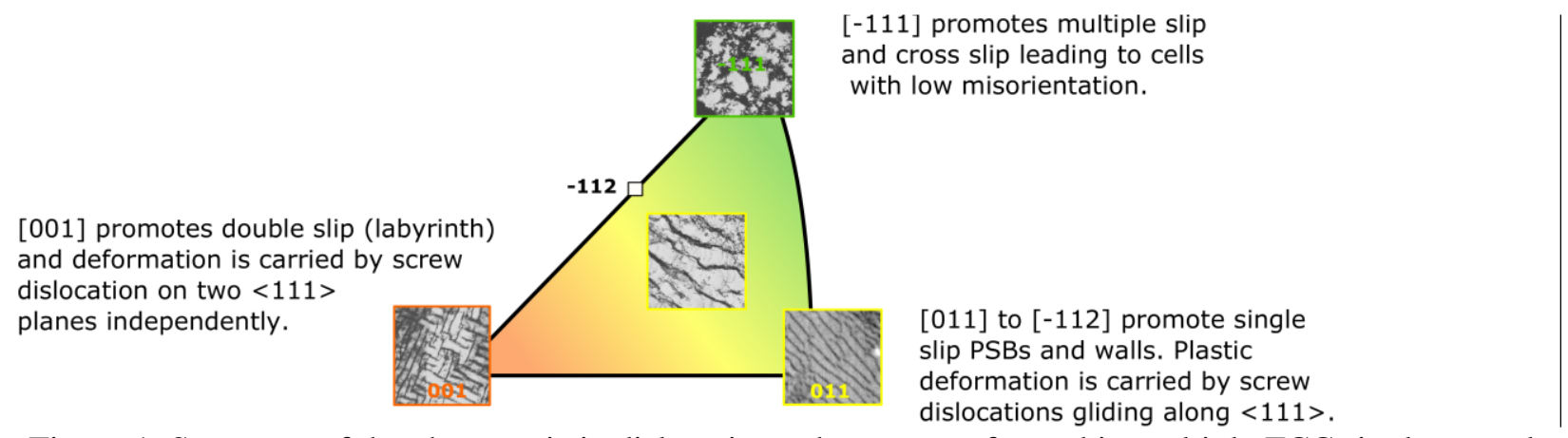

Figure 1. Summary of the characteristic dislocation substructures formed in multiple FCC single crystals after uniaxial cyclic loading along multiple orientations (TEM from Argon, 1975).

The study of single crystals provides a means to link dislocation substructures to the ranges of resolved shear stress and cyclic plastic shear strain associated with each slip system. Currently, no theoretical framework can truly predict the structure that will dominate under cyclic loading, and there are concerns if it will be ever possible to approach the problem exclusively via bottom-up modeling strategies (Ananthakrishna, 2007). Although dislocation dynamics may provide some assistance in understanding the origin of the structures, it is computationally demanding and has not systematically predicted such structures, especially under cyclic loading.

Instead, we adopt an approach based on the experimental fact that only a few well defined structures develop. Li et al. (Li et al., 2011) and the references therein, showed that many FCC metals with a wide range of stacking fault energies ( $\mathrm{Au}, \mathrm{Cu}, \mathrm{Ni}$, and austenitic stainless steels) present a few similar dislocation patterns: vein patches, PSBs, labyrinth, organized walls, and cells. Such similarities support the notion that the same mechanisms are operative among many FCC metals.

To seek correlations between loading orientation and resulting dominant dislocation structure, we consider the relative degree of activation of different slip systems. A mapping based on expected slip system activation is essential in order to address multiaxial strain state effects. Figure 2 presents normalized 3 | P a g e 
projections of the stress along several crystallographic directions for uniaxial loading. This Figure considers:

a) Maximum Schmid factor (SF) for the slip system with the highest resolved shear stress $\left(\tau_{\max }^{\alpha}\right)$,

b) SF for the slip system with the second highest resolved shear stress,

c) The so-called $\mathrm{Q}$ factor, which is the ratio of (b) to (a),

d) The relative SF of the slip system that can cross slip $\left(\tau_{C S_{\max }}^{\alpha}\right)$ from the plane of $\tau_{\max }^{\alpha}$ in FCC metals, defined by

$$
S F^{C S}=\frac{\tau_{C S_{\max }^{\alpha}}^{\alpha}}{\tau_{\max }^{\alpha}}
$$

e) The normalized maximum SF among slip systems that form Hirth locks in FCC metals to the maximum Schmid factor, defined by

$$
S F^{H L_{\max }}=\max \left(\frac{\tau_{H L_{\max }}^{\alpha}}{\tau_{\text {max }}^{\alpha}}\right)
$$

According to the $\mathrm{Q}$ factor plot in Figure 2(c), loading orientations in the central portion of the inverse pole figure will promote deformation under single slip. In such a region, the $\mathrm{Q}$ factor is less than about 0.9, which usually corresponds to single slip (Cheng and Laird, 1981; Sauzay et al., 2014) and would most likely develop PSBs. Based on the $S F^{C S}$ contours in Figure 2(d), cross slip is inhibited in the blue region. Since the formation of cell structures is strongly associated with cross slip events, cells would not easily form for crystals loaded along these orientations. Finally, Figure 2(e) presents the $S F^{H L_{\max }}$; Hirth locks are usually associated with labyrinth structures, and the uniaxial loading in the red orientations is more likely to promote these structures.

a)

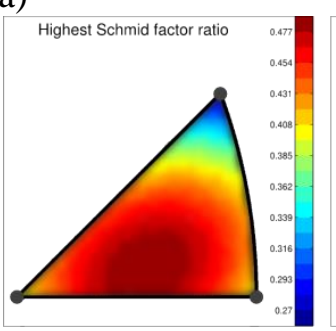

b)

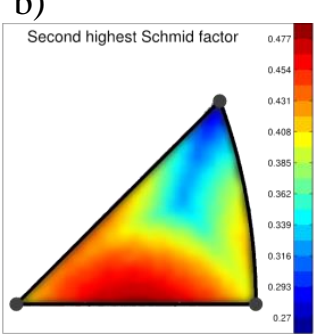

c)

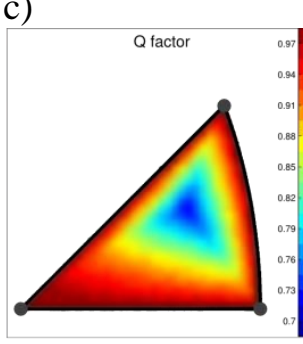

d)

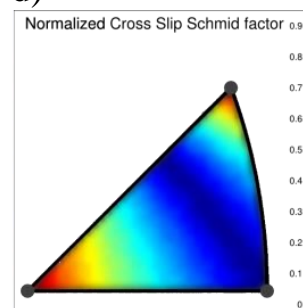

e)

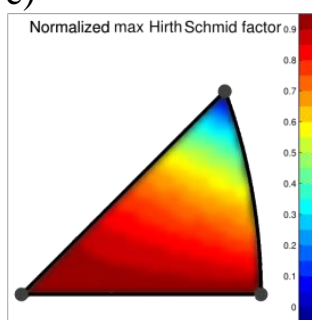

Figure 2. Dependence on the uniaxial loading direction relative to crystal orientation for (a) the maximum SF, (b) second highest SF, (c) Q factor, (d) normalized cross slip plane SF and (e) normalized maximum Hirth factor.

Assuming that only a finite number of mesoscale structures can form (i.e., cells, labyrinth, PSBs, and veins), the challenge is to find a mathematical function or a set of rules that predict the dominant structure for an arbitrary loading conditions. Since we seek to describe the saturated cyclically stable state for each loading amplitude, these rules should satisfy the following criteria motivated from experimental evidence:

- Metallic materials with medium to high stable SFE (e.g., Ag, Cu or Ni) allow for cross slip to occur at low uniaxial stresses and develop walls formed by edge-dominated dislocation dipoles with different geometries. Cells form more easily with increasing SFE. 
- Metallic materials with low stacking fault energies (e.g., Brass) under low applied stresses activate little cross slip and usually form planar pileups, stacking faults and martensitic phases; this is not the case for medium to high SFE FCC metals and will not be considered in this paper.

- Single crystals oriented for single slip (e.g., uniaxial loading along the [-123], [153], and [149] directions) typically form ladder-type walls. This case leads to the formation of PSBs and a clear plateau in the cyclic stress-strain curve (CSSC), as depicted in Figure 3. As shown by Mughrabi (Mughrabi, 1979), if the cyclic plastic shear strain range is below $\Delta \gamma_{M}^{p l}$ (i.e., very high cycle fatigue (VHCF) regime), edge dislocations form veins and patches. For cyclic plastic shear strain ranges above $\Delta \gamma_{P S B}^{p l}$ (low cycle fatigue), cell structures are formed.

- Uniaxial loading along the [001] direction tends to form walls of labyrinth (maze) configuration due to the activation of multiple slip systems. At low cyclic plastic shear strains, veins and patches form. With increasing cyclic plastic strain amplitude, labyrinths and later cells form and the CSSC does not show a significant plateau. Wang et al. (Wang et al., 1997) analyzed $\mathrm{Cu}$ deformation and found the lack of a plateau is associated with labyrinth structures, and that screw dislocations (parallel to $\langle 111\rangle$ ) glide between the walls, which are often parallel to the $\langle 100\rangle$ direction; other wall orientations such as $\langle 210\rangle$ also have been observed.

- Crystals cyclically loaded along the [011] direction typically form walls comprised of combinations of ladder-type structures. Similar to the single slip case, the CSSC tends to present a plateau approximately in the range of $\Delta \gamma_{M}^{p l}$ to $\Delta \gamma_{P S B}^{p l}$. Both the [001] and [011] uniaxial loading directions result in multiple active slip systems with a SF of 0.41, but the SF on the cross slip systems is 0.41 for the former, and zero for the latter; therefore, the [011] direction does not promote cross slip.

- The [111] loading direction has the lowest possible SF and results in formation of cells. The CSSC does not usually present a plateau in this case and can be differentiated from loading along the [001] direction by the activation of Hirth locks in the latter case.

- For all loading directions, if the peak stress or strain increases sufficiently, multiple slip systems activate and cell structures will form. Therefore, increasing the stress ratio (R) or the mean stress leads to cells regardless of the loading direction (Lukas et al., 1999) due to an increase in cross slip activity. 


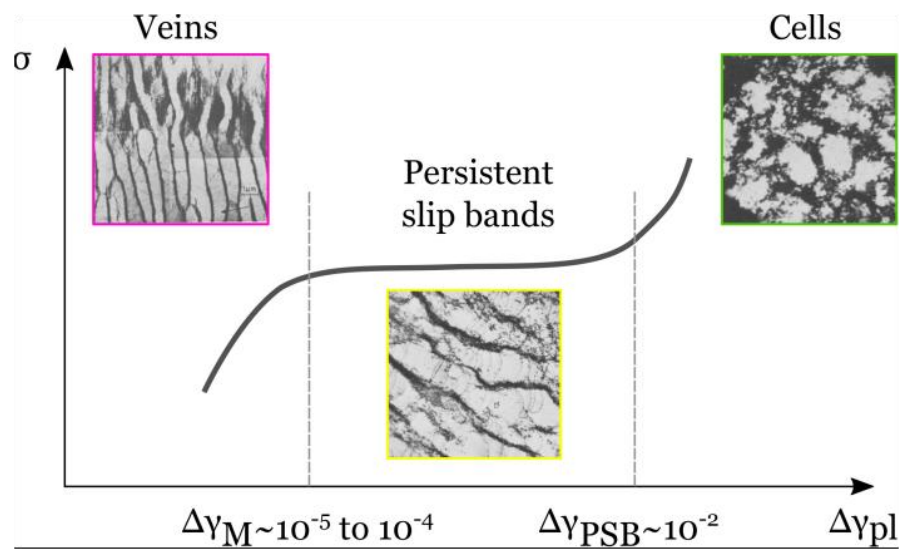

Figure 3. Cyclic stress-strain curves for $\mathrm{Cu}$ single crystals oriented for single slip and the corresponding dislocation substructures that arise at different strain levels (TEM from Argon, 1975).

Understanding the linkage between the factors plotted in Figure 2 based on single crystal experiments under uniaxial loading can be improved, for example, by application of machine learning algorithms rooted in modern data science approaches, assuming a sufficiently large database exists. These rules should also comply with dislocation mechanics, which adds complexity. For the sake of simplicity, we assume that the dominant dislocation substructure at each finite element integration point follows the logic of the strainbased logical flowchart or decision tree shown in Figure 4. To generalize beyond simple uniaxial loading conditions, the logic is based on the local cyclic plastic slip activity and the relative activity on various slip systems. This scheme projects the dominant expected dislocation structure by assessing the local range of plastic shear strain over the last loading cycle $\left(\Delta \gamma^{\alpha}\right)$ among different slip systems, $\alpha$ as follows:

- Single crystals oriented for activating a single dominant slip system develop ladder-type PSBs for cyclic plastic shear strain ranges between $\Delta \gamma_{M}^{p l} \sim 6 \times 10^{-5}$ to $\Delta \gamma_{P S B}^{p l} \sim 1 \times 10^{-2}$. These values are consistent among various FCC metals (Mughrabi, 1979) and we regard them as intrinsic kinematic attributes that weight the activation of secondary and cross slip. Furthermore, veins develop with the maximum cyclic plastic shear strain amplitudes at the slip system level, $\Delta \gamma^{\max }$ , less than $\Delta \gamma_{M}^{p l}$.

- If the highest cyclic plastic shear strain amplitude among all slip systems exceeds $\Delta \gamma_{P S B}^{p l}$, we assume that multiple slip occurs and cells may form. Furthermore, cells would appear for low strain ranges associated with Hirth locks (towards [111] loading in Figure 2(e)). If Hirth locks form on the most active slip system ( $\Delta \gamma^{\text {Hirth }}$ ), labyrinth structures are more prone to appear ( $\mathrm{Li}$ et al., 2011).

- Each substructure has a characteristic distribution of spacing between walls $\left(d_{\text {struct }}\right)$ and structure length $\left(l_{\text {struct }}\right) ; l_{\text {struct }}$ corresponds to the distance between walls along the dominant slip system direction). The ratio of these distances, $\eta=l_{\text {struct }} / d_{\text {struct }}$, can be much greater than unity in PSBs. Although dislocation structures tend to have similar spacing at equivalent shear stress, their mean free paths can differ considerably. Indeed, screw dislocations can glide over significantly longer distances in PSBs than in cells with the same $d_{\text {struct }}$.

We emphasize that the flowchart in Figure 4 is intended to apply to the saturated or cyclically stable state for various FCC metals with medium to high SFE. The selection of the structure depends on the local 
crystallographic strain range over one loading cycle and we are not limited to evaluating uniaxially loaded single crystals.

Cyclic saturation requires a few hundred or thousand cycles, and is a relatively small fraction of total lifetime in most engineering fatigue life assessments for crack tip or notch root applications. Furthermore, it is likely that a crack cyclic plastic zone is almost always at or near saturation and satisfies mesoscale similitude, which means that upon identical fatigue driving forces and crystal orientation, similar mesoscale structures develop at the crack tip regardless of the crack length. Cyclic loading of smooth specimens may not satisfy this requirement at relatively low cyclic strain amplitudes (or overloads/underloads) and would require strategies to assess transient evolution of dislocation structures.

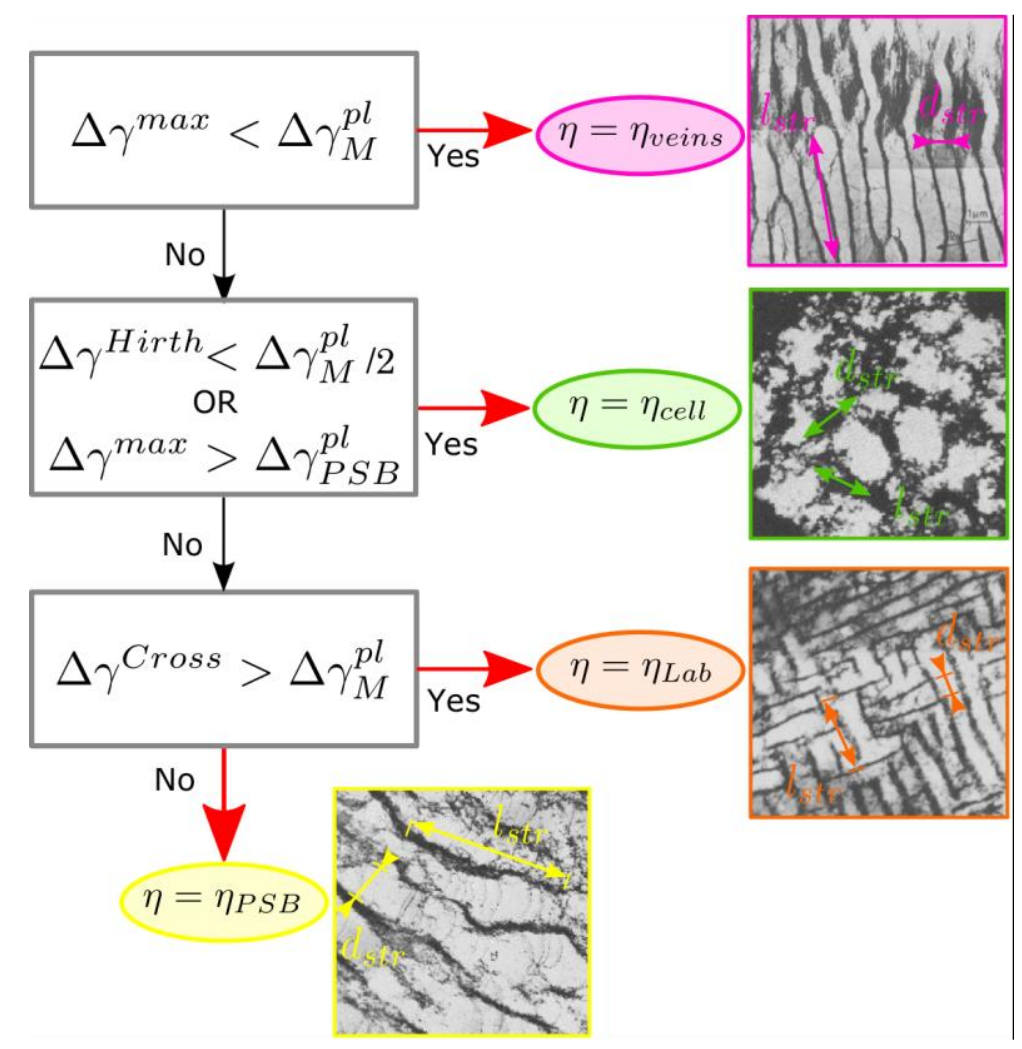

Figure 4. Proposed flowchart for assigning cyclically saturated dislocation substructures for a given state of cyclic plastic shear among slip systems within a crystal associated with a finite volume, for example an integration point of a finite element implementation. The parameter $\eta$ corresponds to the ratio between

$l_{\text {struct }}$ and $d_{\text {struct }}$, which are schematically depicted on the micrographs (TEM from Argon, 1975).

\section{Dislocation evolution}

In most FCC metals, after dislocation structures and stress stabilize during cyclic loading, plastic deformation is dominated by the generation and glide of screw dislocations in between dense dislocation walls comprised largely of dislocations loops with dominant edge components. Therefore, following Kocks et al. (Kocks et al., 1975), we argue that the average shearing rate in-between obstacles depends only on the density of mobile screw dislocations, i.e.,

$$
\dot{\gamma}_{0}=\rho_{m}^{s} \bar{l} b v_{G},
$$


in which $\rho_{m}^{s}$ is the density of mobile (screw) dislocations, $\bar{l}$ is the mean advance distance of a dislocation in between obstacle bypass events, $b$ is the Burgers vector $\sim 2.5^{-10} \mathrm{~m}$, and $v_{G}$ is an estimate of the attempt frequency $\sim 10^{12} \mathrm{~s}^{-1}$.

Since $\dot{\gamma}_{0}$ pre-multiplies an exponential function, the factors in $\dot{\gamma}_{0}$ have a secondary impact on the macroscopic behavior and only an order of magnitude estimate is adequate. Equation (6) refers to mobile dislocations, which are assumed to have screw character in saturated conditions and are rate limiting carriers of deformation. Mesoscale dislocation structures are populated with much higher densities of edge dislocation dipoles, which can be idealized as elastic structures. We may presume that only at very high stresses may these structures contribute substantially to cyclic plastic shear; even in this case, the recovery of the dislocations in walls by annihilation (i.e., wall thinning) may preclude any significant plastic deformation of walls.

The mean free path for dislocation glide, $\bar{l}$, depends on applied stress range and crystal orientation. With increasing applied stress, more dislocations are generated which further reduce the mean free path of dislocations. This phenomenological behavior has commonly been incorporated into constitutive models by assuming that the mean free path inversely depends on the mean dislocation density, i.e.,

$$
\bar{l} \approx 1 / \sqrt{\rho_{m}^{s}}
$$

However, this is a macroscopic simplification that is not consistent with fundamental bottom-up arguments (Langer, 2015). In general, $\bar{l}$ is most likely anisotropic and differs for loading in various crystal orientations.

Given the lack of a fundamental approach to determine a valid statistically representation of the length scales that control work hardening in cyclic plasticity, we propose a geometric model related to dislocation structure configurations that follow from the flowchart in Figure 4. The mean advance distance is determined by the appropriate dislocation mesoscale substructures and not by dislocation density as suggested by Equation (7). Also, the spacing of dislocation walls $\left(d_{\text {struct }}\right)$ follows similitude (KuhlmannWilsdorf, 1962; Sauzay and Kubin, 2011) and typically ranges from $100 \mathrm{~nm}$ to $2 \mu \mathrm{m}$, depending on the inverse of the macroscopic applied stress. Screw dislocations glide in between these walls with transit distance that depends on the morphology of the walls (e.g., cells, PSBs). Therefore, we propose a refinement of Equation (7), i.e.,

$$
\bar{l} \approx l_{\text {struct }}=\eta d_{\text {struct }}
$$

in which $d_{\text {struct }}$ is considered as the characteristic dislocation substructure length scale. These magnitudes are graphically defined for different structures in Figure 4. In addition, Figure 5 depicts a schematic example of a dislocation substructure formed by parallel walls of edge dislocations, between which dominantly screw dislocation segments bow out, glide and pile-up. The distances represented in this Figure 5 can be measured with microscopy techniques with an error on the order of the width of the dislocation walls, but precise definition and quantification of these magnitudes is outside the scope of the present work. 

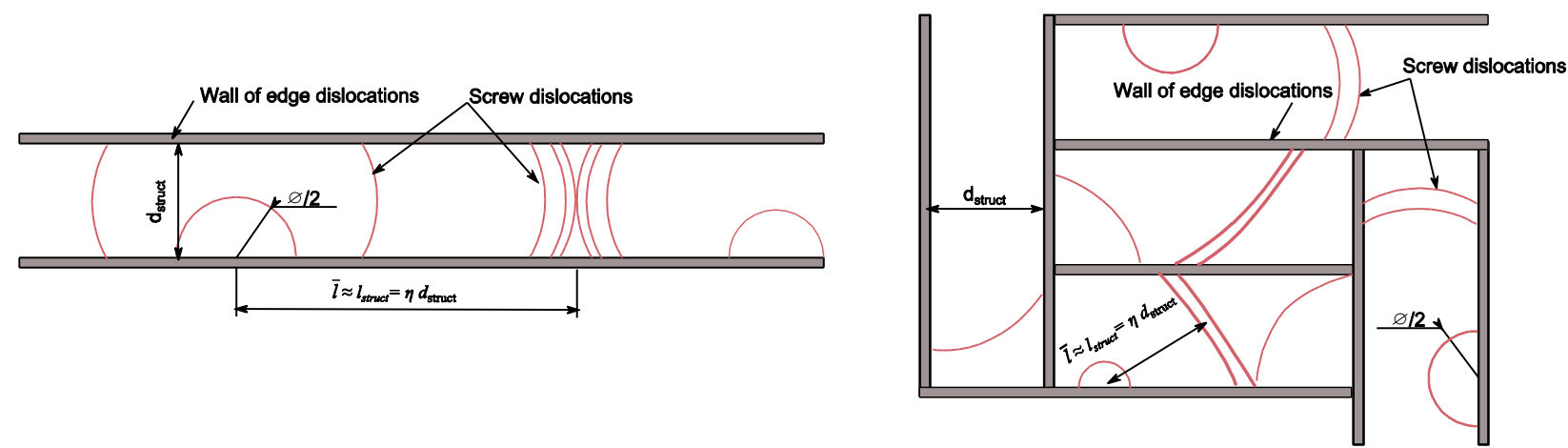

Figure 5. Schematic representation of a dislocation bowing out from a dislocation wall, contributing to extended screw dislocation glide and dislocation pileups in PSBs and labyrinth structures.

The factor $\eta$ reflects the normalized distance that a dislocation can glide along the dominant slip system relative to the distance between walls. A limited survey of TEM experiments (cf. Figure 4) suggests that $\eta_{\text {cell }} \sim 1$ for cells, but for PSBs $\eta_{P S B} \sim 5-25$. In fact $\eta_{P S B}$ has a much larger intrinsic error than $\eta_{\text {cell }}$ since $\bar{l}$ has the potential to span the entire grain cross section in PSBs, especially for initial low dislocation densities and/or large grain sizes. We highlight that these magnitudes are not fitting constants, but rather are stochastic parameters that should be validated with a statistical characterization of TEM images (e.g., Oudriss and Feaugas, 2016) or dislocation dynamics modeling.

Our characterization of the structures assumes that the matrix between walls is effectively depleted from dislocations. Thus, once a dislocation bows out, it will glide with single slip behavior until it reaches the structure boundary. This mechanism is valid even for cells, in which a single slip system is active at a time inside each cell, but different systems may be activated at different loading levels or within neighboring cells. Thus, "hard" dislocation locks may only form in walls rather than in regions of easy glide. Such a characterization agrees with energetic considerations that single slip corresponds to an energy well and later hardening arise from the development of mesoscale structures (Conti and Ortiz, 2005; Hansen et al., 2010; Ortiz and Repetto, 1999).

Another characteristic distance associated with the dislocation structure is the wall thickness, which along with the structure spacing and length determines the wall volume fraction, $f_{w}$. The experimental quantification of the size of the wall is difficult and varies among multiple authors for two main reasons:

1. TEM data from various loading conditions is required to compute spacing and thicknesses of walls for single crystals (that allow the quantification of the plastic shear strain range).

2. The calculation of $f_{w}$ assumes a geometric configuration of walls that is subject to various assumptions and idealizations.

In general, $f_{w}$ ranges between 0.1 to 0.7 , depending on the stress and strain, but is almost independent of either temperature or the number of cycles after saturation (Pham and Holdsworth, 2014). Furthermore, the wall volume fraction decreases with increasing plastic strain range as a result of dynamic recovery processes that thins the walls. Recovery is usually associated with the annihilation of dislocations, but quantitative models are lacking to relate these processes to the thinning of dislocation walls. Furthermore, the wall thickness does not follow similitude scaling relation (Sauzay and Kubin, 2011). Thus, we employ a phenomenological parametrization of $f_{w}$ based on the maximum plastic shear strain range per cycle among all slip systems, which resembles the approach by Estrin et al. (Estrin et al., 1998), i.e.,

$$
f_{w}=f_{\text {inf }}+\left(f_{0}-f_{\text {inf }}\right) \exp \left(\frac{-\Delta \gamma^{\max } / 2}{g_{p}}\right)
$$


in which $f_{\text {inf }}, f_{0}$, and $g_{p}$ are material constants that can be estimated by computing $f_{w}$ from TEM images. Naturally, we would prefer a bottom-up scheme that quantifies the evolution of the wall thickness, but this is currently a grand challenge. Certainly, the problem is not even amenable to application of current discrete dislocation dynamics approaches due to the large number of cycles and strain level required. Despite these difficulties, we cannot neglect effects of these structures on the mechanical response so we will rely on mesoscale parametrization.

The dislocation substructure length scale, $d_{\text {struct }}$, represents the minimum distance between dislocation walls and follows the similitude scaling law (Sauzay and Kubin, 2011) inversely related to the maximum shear stress, i.e.,

$$
d_{\text {struct }}=\frac{K_{\text {struct }} \mu(T) b}{\tau} .
$$

Sauzay and Kubin (Sauzay and Kubin, 2011) discussed in detail the validity of similitude relations of this type under monotonic and cyclic conditions, and whether the wall thickness is included in Equation (10); here we assume that the error of this equation is on the order of the thickness of the wall. Also, they showed that constant $K_{\text {struct }}$ ranges between 3 to 4 for a large number of metals and a wide range of cyclic loading conditions.

Our modeling approach employs Equation (10) to estimate the dislocation substructure dimension for each finite element integration point and considers the maximum shear stress over the last loading cycle among all slip systems $\left(\tau=\tau_{\max }^{\alpha}\right)$. Since $d_{\text {struct }}$ is assumed to follow similitude, $l_{\text {struct }}$ also has such a property, which has also been proposed to be true under certain loading conditions (Zaiser and Sandfeld, 2014). Although mesoscale parameters (e.g., $\eta, f_{w}, K_{\text {struct }}$ ) can exhibit significant variability that depends on the loading direction and history, they can be computed and bounded with experimental data. More importantly, these magnitudes tend to be similar across FCC systems, facilitating application of common algorithms for uncertainty quantification among metallic materials.

\section{Misorientation and mesoscale dislocation substructure distributions}

Multiple experimental efforts conducted under monotonic and cyclic loading conditions have shown that local crystallographic misorientation within grains increases with increasing plastic strain. For example, Kamaya and Kuroda (Kamaya and Kuroda, 2011) quantified the misorientation distribution of dislocation substructures for stainless steel 316 under cyclic deformation, as shown in Figure 6.

Crystal plasticity models present difficulties in predicting precise local misorientation associated with subgrains and dislocation wall structures. This is usually due to two main reasons: a lack of localization of lattice rotation at the scale of mesoscale structures (which may contain polar dislocation distributions) and the incorrect partition of plastic deformation among slip systems. The latter affects the competition between elastic stored energy and dissipated energy and usually leads to a reduction in relative lattice rotation by virtue of activating additional slip systems. Under single slip deformation, secondary slip is greatly reduced and crystal plasticity has a better chance to capture the magnitude of experimentally observed local lattice rotations (Lloyd, 2010). Lattice rotations are significantly reduced relative to the single slip case when multiple slip systems are activated, such as in single crystals loaded along [001] or [111] orientations. 


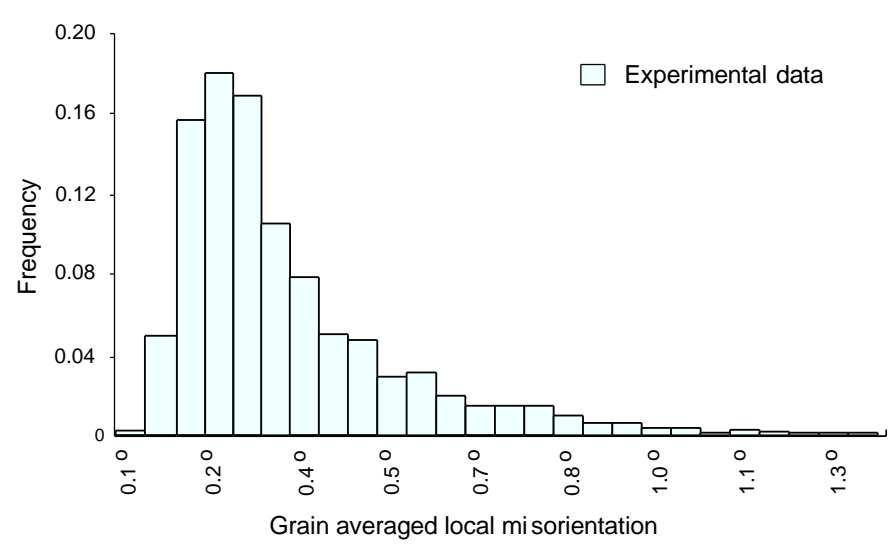

Figure 6. Frequency distribution of grain-averaged local misorientation for $1 \%$ cyclic strain amplitude at $80 \%$ of the fatigue life for stainless steel 316 (Kamaya and Kuroda, 2011).

Unless mesoscale localization is explicitly resolved (e.g., explicitly modeling localization of dislocation density), local rotations induced by polar densities should be introduced as an external artifact induced by stochastic processes involving mesoscale structures. Therefore, we follow the parametrization proposed by Hughes (Hughes et al., 1997, 1998), who assessed misorientation distributions among subgrains for various FCC metals with medium to high SFE subjected to monotonic plastic deformation. They proposed that the misorientation probability density function satisfies the relation,

$$
\Delta \theta_{a v} p(\Delta \theta)=\frac{\alpha^{\alpha}}{\Gamma(\alpha)}\left(\frac{\Delta \theta}{\Delta \theta_{a v}}\right)^{\alpha-1} \exp \left(-\frac{\alpha \Delta \theta}{\Delta \theta_{a v}}\right),
$$

where $p(\Delta \theta)$ is the probability density function for a misorientation equal to $\Delta \theta, \Delta \theta_{a v}$ is the average misorientation measured at each strain level, and $\Gamma(\alpha)$ corresponds to the gamma function with $\alpha$ approximately two to three.

The average misorientation $\Delta \theta_{a v}$ is a function of the loading history and it has been correlated to the applied strain level in shear or compression experiments. For monotonic loading, several authors (Hughes et al., 1997) proposed power law dependence on the strain; the power depends on the dislocation structures. Here, we consider dependence on the effective plastic deformation $\varepsilon_{\text {eff }}^{p}$, e.g.,

$$
\Delta \theta_{a v} \approx K_{M C D}\left(\varepsilon_{\text {eff }}^{p}\right)^{1 / 2},
$$

which should be regarded as an approximation pending further validation, since the exponent may vary with the substructure [26].

The asymmetric misorientation distribution is characteristic and deeply connected to the statistics of dislocation interactions. For example, Cleri (Cleri, 2005) employed stochastic dynamics to study dislocation patterns and showed that the distribution of dislocation cell structures can be associated with statistical orientation fluctuations that follow Equation (11). Such fluctuations are due to the random dislocation trapping at cell boundaries, which can be considered as a stochastic process.

Misorientation distributions can be introduced in crystal plasticity by adding a stochastic perturbation to each of the Euler angles that speficy the lattice orientation. Therefore, by integrating Equation (11) we compute the misorientation probability, i.e., 


$$
\int_{0}^{\partial \theta} p(\Delta \theta)=\int_{0}^{\partial \theta} \frac{\alpha^{\alpha}}{\Delta \theta_{a v} \Gamma(\alpha)}\left(\frac{\Delta \theta}{\Delta \theta_{a v}}\right)^{\alpha-1} \exp \left(-\frac{\alpha \Delta \theta}{\Delta \theta_{a v}}\right)=\frac{1}{\Gamma(\alpha)} \int_{0}^{\partial t} \frac{\Delta \theta_{a v}}{\alpha}(t)^{\alpha-1} \exp (-t) d t
$$

which yields a function of the lower incomplete gamma function, i.e.,

$$
P(\Delta \theta)=\gamma\left(\alpha, \frac{\alpha \Delta \theta}{\Delta \theta_{a v}}\right)
$$

Inverting this function and taking into account Equation (12) we obtain

$$
\Delta \theta_{\varphi_{1}, \phi, \varphi_{2}}=K_{M C D}\left(\varepsilon_{e f f}^{p}\right)^{1 / 2} \gamma^{-1}(x, \alpha),
$$

in which $\boldsymbol{\gamma}^{-1}$ is the inverse lower incomplete gamma function for a random value of $x$ between 0 and 1 , $\alpha=3$. The scaling parameter $K_{M C D}$ represents the rotation irreversibility and can be estimated from experiments (e.g., Figure 6) or dislocation dynamics simulations. Note that the random variable $x$ is computed at each integration point in a finite element implementation only once at the beginning of a simulation; it is kept constant throughout the cyclic loading for a given loading condition. The angles $\Delta \theta_{\varphi_{1}, \phi, \varphi_{2}}$ in Equation (15) correspond to the perturbation on the lattice orientation induced by the mesoscale and it is added to the current Euler angles for that orientation.

The localization of dislocations into walls form patterns with characteristic length scales that depend on the loading level, history and crystal orientation. Figure 4 presents a deterministic approach for estimating the structure length scales, which would correspond to the mean or most likely scenario. To consider intrinsic variability in these structures, we introduce normal distributions for $d_{\text {struct }}, f_{w}$ and $\eta_{\text {struct }}$ centered at the mean values predicted from the deterministic calculation. For example,

$$
d_{\text {struct }}=d_{\text {struct }}^{0} \frac{1}{2}\left(1+\operatorname{erf}\left(\frac{x-1}{\sqrt{2} S D}\right)\right)
$$

in which $d_{\text {struct }}^{0}$ is the deterministic value predicted with Equation (10) and $\frac{1}{2}\left(1+\operatorname{erf}\left(\frac{x-1}{\sqrt{2} S D}\right)\right)$ employs the error function, $\operatorname{erf}()$, to compute the cumulative normal distribution function with mean value of 1 and standard deviation, SD, provisionally estimated as 0.1 . The shape of the distribution for cyclic loading is unknown, but for monotonic loading Feaugas and coworkers (Oudriss and Feaugas, 2016) have recently characterized them as asymmetric using an approach similar to Equation (16) with an SD of 0.1. A detailed experimental characterization of the distribution may help improve estimates of stresses and strains, but it probably has only a secondary influence on the homogenized macroscopic behavior.

\section{Dislocation density rate: multiplication, annihilation and cross slip}

Following the assumption that only screw dislocations are mobile, we propose that the mobile dislocation density on slip system $\alpha$ results from a balance between multiplication, annihilation and cross slip (Arsenlis and Parks, 2002; Essmann and Mughrabi, 1979; Patra and McDowell, 2012), i.e.,

$$
\dot{\rho}_{m}^{s \alpha}=\dot{\rho}_{m u l t}^{s \alpha}-\dot{\rho}_{\text {annih }}^{s \alpha}+\left.\dot{\rho}_{c s}^{s}\right|^{\zeta \rightarrow \alpha}-\left.\dot{\rho}_{c s}^{s}\right|^{\alpha \rightarrow \zeta}
$$

The multiplication rate of mobile screw dislocations for slip system $\alpha$ is computed as

12 | P a g e 


$$
\dot{\rho}_{\text {mult }}^{s \alpha}=\frac{k_{\text {multi }}}{b l_{\text {struct }}}\left|\dot{\gamma}^{\alpha}\right|
$$

Equation (18) parameterizes the homogenized response of various dislocation arrangements, while $k_{\text {multi }}$ regulates the production of dislocations. As shown in Figure 7, we assume that two geometrical arrangements dominate the production of dislocations at the saturated cyclic state: a single dislocation bows outs cell walls (Tabata et al., 1982) while two screw dislocations bow out from parallel walls (Essmann and Mughrabi, 1979); hence, $k_{\text {multi }}=1$ for cells and $k_{\text {multi }}=2$ for parallel walls such as PSBs/labyrinth. We envision that discrete dislocation dynamics can play an important role in providing more realistic parametrizations of $k_{\text {multi }}$ for different internal state variables and loading conditions.
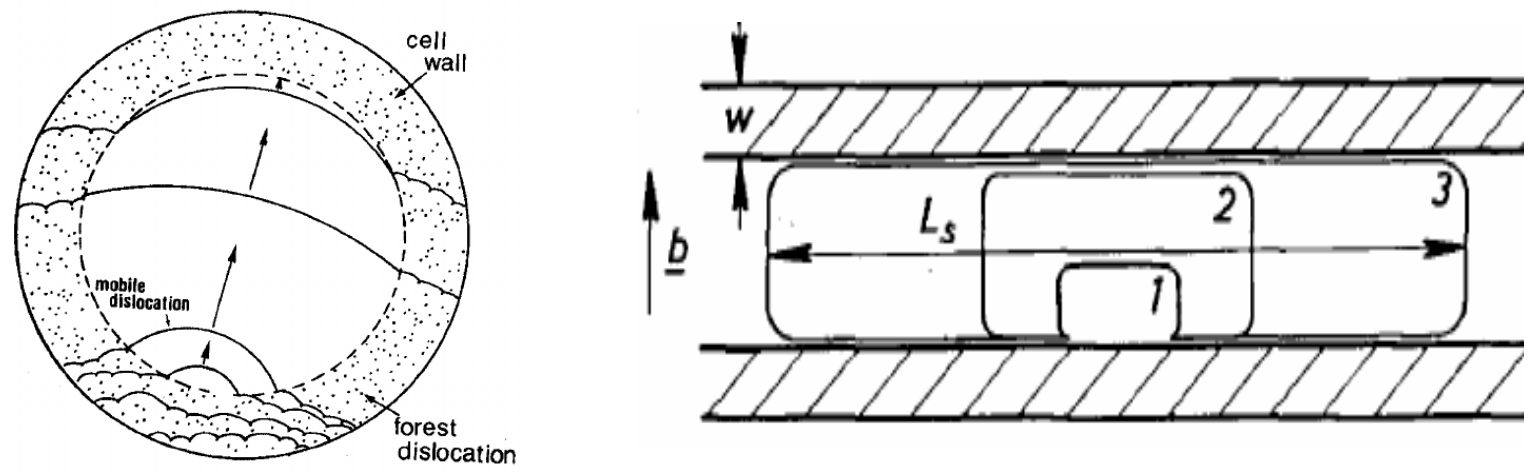

Figure 7. Schematic representation (at left) of a mobile dislocation inside a cell (from Tabata et al., 1982), and schematic multiplication (at right) via two screw dislocations gliding within the channel within parallel walls (from Essmann and Mughrabi, 1979).

We propose that the annihilation rate of mobile screw dislocations is given by

$$
\dot{\rho}_{\text {annih }}^{s}{ }^{\alpha}=\frac{2 y_{s}^{\text {edge }}}{b} \rho_{m}^{s \alpha}\left|\dot{\gamma}^{\alpha}\right|-\left.\Upsilon \frac{2\left|\dot{\tau}^{\alpha}-\dot{B}^{\alpha}\right|}{\mu b d_{\text {struct }}}\right|^{\text {Unload }},
$$

where $\Upsilon=1$ if $\operatorname{sgn}\left(\dot{\tau}^{\alpha}-\dot{B}^{\alpha}\right) \neq \operatorname{sgn}\left(\tau^{\alpha}-B^{\alpha}\right)$ or $\dot{\gamma}^{\alpha}=0$; otherwise $\Upsilon=0$ and $\left.\dot{\rho}_{\text {annih }}^{s}\right|^{\text {Unload }}=0$. The first term considers the annihilation mechanisms that involve plastic deformation without cross slip (e.g., edge dislocation annihilation), for which the annihilation distance, $y_{s}^{\text {edge }}$, has a value in the range of 0.5 - 5 $\mathrm{nm}$ for pure FCC metals (Aslanides and Pontikis, 2000). Cross slip annihilation is taken into account in the last term on the right hand side of Equation (17) and is not considered in $\dot{\rho}_{\text {annih }}^{s}{ }^{\alpha}$ defined in Equation (19). Approaches that consider a single annihilation distance based on a cross slip mechanism can significantly overestimate the annihilation for single crystals under single slip conditions since there is insufficient driving force for dislocations to cross slip.

The second term in Equation (19) is non-standard and represents the annihilation of dislocations due to anelastic deformation upon reversing the local shear stress. This process corresponds to the reversible production and subsequent annihilation of dislocation, and it is readily observed as anelastic dislocation annihilation (reduction in dislocation density without net plastic deformation), which typically occurs in the quasi-elastic response immediately following a load reversal. Various authors have attempted to quantify this effect with different approaches. Estrin et al. (Estrin et al., 1996) introduced such an additional annihilation effect in dislocation density evolution models by resetting the amount of recoverable immobile

13 | P a g e 
dislocations for every load reversal. Wen and coworkers (Wen et al., 2016) proposed an empirical formulation to quantify the mechanical reversibility of dislocation production by introducing an additional annealing of dislocations during cyclic loading.

Our approach models the unloading annihilation process by assuming that two dislocation pileups of opposite sign annihilate during unloading. Following Cottrell (Cottrell, 1953), the number of dislocations in a pileup of length $L$ can be computed as

$$
n \approx \frac{\pi L \tau}{\mu b}
$$

These dislocations have a length on the order of $d_{\text {struct }}$ (because they are constrained by walls) and transit between wall structures within a volume proportional to $d_{\text {struct }} \times d_{\text {struct }} \times \eta d_{\text {struct }}$. Therefore, assuming that $L \approx l_{\text {struct }}=\eta d_{\text {struct }}$, the dislocation density can be approximated as

$$
\rho \approx \frac{\pi \tau}{\mu b d_{\text {struct }}}
$$

Taking the derivative with respect to time and acknowledging that this annihilation mechanism becomes active during purely elastic unloading, i.e.,

$$
\left.\dot{\rho}_{\text {annih }}^{s}{ }^{\alpha}\right|^{\text {Unload }}=\left.\Upsilon \frac{2\left|\dot{\tau}^{\alpha}-\dot{B}^{\alpha}\right|}{\mu b d_{\text {struct }}}\right|^{\text {Unload }}
$$

Further, we interpret the case $\rho^{\alpha}<\left(\frac{1}{d_{\text {struct }}}\right)^{2}$ as corresponding to complete annihilation of dislocation pileups within mesoscale dislocation walls and we assume $\Upsilon=0$ for such a case. Note that the local shear stress rate $\left(\dot{\tau}^{\alpha}-\dot{B}^{\alpha}\right)$ controls the annihilation rate.

The rate of cross slip of mobile screw dislocations from plane $\alpha$ to $\zeta\left(\left.\dot{\rho}_{c s}^{s}\right|^{\alpha \rightarrow \zeta}\right)$ is estimated from the probabilistic formulation proposed by several researchers (Kubin et al., 1992; Verdier et al., 1998; Zhou et al., 2010), but considering the local shear stress to account for shielding effects from dislocation structures, i.e.,

$$
\dot{\rho}_{c s}^{s \alpha}=-v_{G} \frac{l_{C S}}{d_{0}} \rho_{m}^{s \alpha} e^{\left(-V_{C S} \frac{\tau_{I I I}-\left|\tau^{\zeta}-B^{\zeta}\right|}{k_{B} T}\right)} .
$$

Here, $v_{G}$ and $l_{C S} \approx d_{\text {struct }}$ are the cross slip characteristic frequency and dislocation length, respectively, $\tau_{I I I}$ is the critical cross slip stress, and $\left|\tau^{\zeta}-B^{\zeta}\right|$ is the magnitude of the local stress on the cross slip plane of $\alpha$. The cross slip activation volume, $V_{C S}$, depends on the dislocation constraints, and its statistical average has been shown to be inversely proportional to the slip system shear stress, i.e.,

$$
V_{C S}^{\alpha}=\frac{V_{C S}^{0}}{\left|\tau^{\alpha}\right|}
$$

with $V_{C S}^{0} \approx 1000 b^{3} M P a$ (Armstrong and Rodriguez, 2006). Other approaches have employed constant activation volumes that range from $20 b^{3}$ to $1800 b^{3}$ for SS316 (Bonneville et al., 1988; Rao et al., 1999; Rasmussen et al., 2000; Ray and Mallik, 1983; Wang et al., 2007). The critical cross slip stress, $\tau_{I I I}$, which 
has been estimated between 30 to 80MPa (Armstrong and Rodriguez, 2006; Mitchell, 1964; Zhou et al., 2010) for $\mathrm{Ni}$, is related to the cyclic shear stress-strain plateau, which is about $50 \mathrm{MPa}$ (Blochwitz and Veit, 1982; Buque, 2001). Here we compute the cross slip stress value following Brown (Brown, 2002), i.e.,

$$
\tau_{I I I}=\frac{\mu b}{4 \pi y_{s}^{\text {screw }}}
$$

where $y_{s}^{\text {screw }}$ corresponds to the annihilation distance of screw dislocations and is controlled by the activation of cross slip.

It is understood that the negative rate contribution in Equation (23) on slip system $\alpha$ for each cross slip system $\checkmark$ is partially compensated by an equal positive rate contribution onto slip system $\varsigma$. This latter contribution is not perfectly efficient, since some of the dislocations that manage to cross slip will annihilate. To account for this inefficiency, a cross slip efficiency parameter $\phi_{C S}$ is introduced. Its value varies from 0 to 1 , and no net change in population of dislocations due to cross slip occurs for $\phi_{C S}=0.5$, which is the expected steady state at saturation.

The dependence of Equation (23) on the local shear stress $\tau^{\alpha}-B^{\alpha}$ rather than on the apparent shear stress $\tau^{\alpha}$ has important implications; if the shear stress $\tau^{\alpha}$ were considered, single crystals oriented for [111] would activate profuse cross slip at lower plastic strains than for [001] orientation. Furthermore, upon an increment of load, most dislocations would cross slip in these highly symmetric loading directions. On the contrary, an increase in $\tau^{\alpha}$ may be accompanied with an increase in back stress, maintaining the local stress $\tau^{\alpha}-B^{\alpha}$ and the cross slip rate. In other words, dislocation substructure has an important influence on cross slip via mediation by the back stress.

In summary, the evolution of screw dislocation density is written as

$$
\begin{aligned}
& \dot{\rho}_{m}^{s \alpha}=\frac{k_{\text {multi }}}{b l_{\text {struct }}}\left|\dot{\gamma}^{\alpha}\right|-\frac{2 y_{s}^{\text {edge }}}{b} \rho_{m}^{s \alpha}\left|\dot{\gamma}^{\alpha}\right|-\left.\frac{2 \pi}{\mu b d_{\text {struct }}}\left|\dot{\tau}_{\text {eff }}\right|\right|^{\text {Unload }}+ \\
& \left.\left.v_{G} \frac{d_{s t r u c t}}{d_{0}}\left(\phi_{C S} \sum_{\zeta=1(\zeta \neq \alpha)}^{N} \rho_{m}^{s \zeta} e^{\left(-V_{C S}^{\alpha} \frac{\frac{\mu b}{4 \pi y_{s}^{\text {screw }}}-\left|\tau^{\alpha}-B^{\alpha}\right|}{k_{B} T}\right.}\right)-(1-\phi) \sum_{\zeta=1(\zeta \neq \alpha)}^{N} \rho_{m}^{s \alpha} e^{\left(-V_{C S}^{\zeta} \frac{\frac{\mu b}{4 \pi y_{s}^{\text {screw }}}-\left|\tau^{\zeta}-B^{\zeta}\right|}{k_{B} T}\right.}\right)\right)
\end{aligned}
$$

for which we have described the origin of each parameter based on physical arguments.

\section{Athermal and threshold stresses}

Dislocation structures affect glide and residual stresses, and assist in promoting local single slip. Within the matrix in a cell or PSB, different mechanisms control the hardening rate, as summarized next.

- Threshold stress $\left(S_{0}^{\alpha}\right)$ : In FCC metals, lattice friction is almost negligible compared to the strength of dislocation interactions and therefore $S_{0}^{\alpha}$ is attributed mainly to the interaction of dislocations with dislocation debris. For completeness the formulation includes a threshold stress $S_{0}^{\alpha} \approx 4 \mathrm{MPa}$ (Mughrabi, 1987; Mughrabi and Pschenitzka, 2005).

- Bow-out stress $\left(\tau_{B o w}\right)$ : Assuming that dislocation multiplication is controlled by the dislocation line energy of bow-outs from walls (depicted by Figure 7), irreversible deformation would not occur before a bow-out reaches the opposite wall. Before this stage, mechanically reversible 
inelastic deformation occurs (though it is thermodynamically irreversible). Thus, the threshold stress that must be attained before plasticity ensues is given by

$$
\tau_{\text {Bow }}=\alpha_{L E} \frac{\mu b}{2 d_{\text {struct }}} .
$$

Here, $\alpha_{L E}$ is a factor that depends on dislocation line energy, typically in the range 0.5 to 1(Tabata et al., 1982). Similar concepts have been proposed for dislocation bow-outs in between precipitates (Tinga et al., 2010; Yuan et al., 1998). Furthermore, Mughrabi and Pschenitzka (Mughrabi and Pschenitzka, 2005) compared the contributions to strength from bow-outs and dipole interactions, and concluded that considering only the former may underestimate the stress by up to $20 \%$. Similar results were found by Jaafar A. El-Awady, 2007.

- Dislocation-dislocation interaction stress $\left(\tau_{D D}^{\alpha}\right)$ between dislocation walls: Under single slip, collinear dislocations interact and form pile-ups. Such an interaction can be captured by considering two opposing pile-ups associated with the same slip system inside the same structure, i.e.,

$$
\tau_{D D}^{\alpha}=\mu b\left(A_{i i} \rho^{\alpha}\right)^{0.5}
$$

Here, $A_{i i}$ corresponds to the self-hardening coefficient from the full interaction matrix (Gerard et al., 2013; Kubin et al., 2008; Schwartz et al., 2013). If we consider the interactions among all dislocation densities of different character: self, collinear, coplanar, glissile, Hirth locks and Lomer locks the results yield excessive hardening. Certainly, the simultaneous interaction of all slip systems does not follow the observed physical picture in which soft cells interiors are depleted of locks and does not correspond to an energy well (Conti and Ortiz, 2005; Hansen et al., 2010; Ortiz and Repetto, 1999).

In summary, the threshold stress required to bow a dislocation against a dislocation pile-up between dislocation walls is given by

$$
S^{\alpha}=S_{0}^{\alpha}+\alpha_{L E} \frac{\mu b}{2 d_{\text {struct }}}+\mu b\left(A_{i i} \rho^{\alpha}\right)^{1 / 2},
$$

which corresponds to a description of latent hardening effects at the dislocation substructure level. Again, we have accounted for the origin of each term and its associated parameters.

\section{Local, effective and macroscopic shear stresses}

Macroscopic stress-strain measurements of the back stress reflect two contributions: the intragranular part $\left(\mathrm{B}_{\text {intra }}\right)$ that originates from dislocation interactions within grains and the intergranular part $\left(\mathrm{B}_{\text {inter }}\right)$ induced by intergranular interactions. The sum of these two contributions can be deduced from cyclic stressstrain data. Pham and coworkers (Pham et al., 2013) considered the back stress in stainless steel and showed that both contributions are significant. In a crystal plasticity approach, intergranular back stress effects are effectively captured by explicitly modeling grains and, as desired, grain boundaries; only the intragranular portion of the back stress $B_{\text {intra }}$ needs to be specified as part of the constitutive framework at the slip system level, i.e., $B^{\alpha}=\mathrm{B}_{\text {intra. }}$.

Sauzay (Sauzay, 2008) employed the Eshelby inclusion formalism (Eshelby, 1957; Kröner, 1961) under the infinitesimal strain assumption and linear elasticity to model back stress induced by the rigidity of areas of high dislocation density, e.g., dislocation walls. Following the work of Berveiller and Zaoui (Berveiller and Zaoui, 1979), he assumed that dislocation walls (hard shell inclusion) constrain the matrix deformation (softer interior of the shell). Furthermore, we showed in a separate work (Hennessey et al., 2017) that this approach results in a hardening-dynamic recovery back stress evolution law similar to those proposed by 
Armstrong-Frederick (Frederick and Armstrong, 2007) or Onho-Wang (Ohno and Wang, 1993). Following a similar spirit, but instead of considering the integrated derivation from Berveiller and Zaoui, 1979, we return to Hill's differential approach (Hill, 1965). Following Berveiller and Zaoui, 1979, we may write

$$
\dot{\boldsymbol{\sigma}}=\dot{\boldsymbol{\sigma}}^{*}+\mathbf{L}^{\prime}\left(\mathbf{S}^{-1}-\mathbf{I}\right)\left(\dot{\boldsymbol{\varepsilon}}^{*}-\dot{\boldsymbol{\varepsilon}}\right)
$$

where $\dot{\sigma}$ and $\dot{\varepsilon}$ are the stress and strain rates in the inclusion, $\dot{\sigma}^{*}$ and $\dot{\boldsymbol{\varepsilon}}^{*}$ are the applied stress and strain rates for the effective medium, and $\mathbf{L}^{\prime}$ and $\mathbf{S}$ are the constraint and Eshelby tensors, respectively.

Decomposing the total strain rate into elastic and plastic contributions, we rewrite this equation as

$$
\dot{\boldsymbol{\sigma}}=\dot{\boldsymbol{\sigma}}^{*}+\mathbf{L}^{\prime}\left(\mathbf{S}^{-1}-\mathbf{I}\right)\left(\dot{\boldsymbol{\varepsilon}}_{p}^{*}-\dot{\boldsymbol{\varepsilon}}_{p}+\dot{\boldsymbol{\varepsilon}}_{e}^{*}-\dot{\boldsymbol{\varepsilon}}_{e}\right)
$$

From elastic considerations (e.g., Khan and Huang, 1995, Equation 11.90), i.e.,

$$
\dot{\boldsymbol{\sigma}}-\dot{\boldsymbol{\sigma}}^{*}=\mathbf{C}\left(\dot{\boldsymbol{\varepsilon}}_{e}-\dot{\boldsymbol{\varepsilon}}_{e}^{*}\right)
$$

we arrive at

$$
\left[\mathbf{I}+\mathbf{L}^{\prime}\left(\mathbf{S}^{-1}-\mathbf{I}\right) \mathbf{C}^{-1}\right]\left(\dot{\boldsymbol{\sigma}}-\dot{\boldsymbol{\sigma}}^{*}\right)=\mathbf{L}^{\prime}\left(\mathbf{S}^{-1}-\mathbf{I}\right)\left(\dot{\boldsymbol{\varepsilon}}_{p}^{*}-\dot{\boldsymbol{\varepsilon}}_{p}\right) .
$$

No exact solution exists for the Eshelby and constraint tensors in Equation (33) for general loading and arbitrary inclusion shape. However, a closed form solution can be found for a spheroidal inclusion under pure shear. Given our intent to develop a back stress evolution law at the slip system level, such a simplification seems adequate. For such a case,

$$
\begin{gathered}
{\left[1+\frac{\mu^{\prime}}{\mu} \frac{1-2 \mathrm{~S}_{1212}}{2 \mathrm{~S}_{1212}}\right]\left(\dot{\tau}-\dot{\tau}^{*}\right)=2 \mu^{\prime} \frac{1-2 \mathrm{~S}_{1212}}{2 \mathrm{~S}_{1212}}\left(\dot{\gamma}^{*}-\dot{\gamma}_{p}\right) .} \\
\dot{\tau}-\dot{\tau}^{*}=\frac{2 \mu^{\prime} \frac{1-2 \mathrm{~S}_{1212}}{2 \mathrm{~S}_{1212}}}{1+\frac{\mu^{\prime}}{\mu} \frac{1-2 \mathrm{~S}_{1212}}{2 \mathrm{~S}_{1212}}}\left(\dot{\gamma}_{p}^{*}-\dot{\gamma}_{p}\right) .
\end{gathered}
$$

in which (Equation 3.11 in Berveiller and Zaoui, 1979)

$$
\mu^{\prime}=\frac{\mu}{1+2 \mu f_{\text {Hill }}}
$$

Here, the Hill factor has been defined as the instantaneous macroscopic plastic deformation tangent, i.e.,

$$
f_{\text {Hill }}=\frac{3}{2} \frac{d \varepsilon_{p}^{*}}{d \sigma^{*}}
$$

Under shear deformation this may be written as

$$
f_{\text {Hill }}^{S}=\frac{1}{2} \frac{d \gamma_{p}^{*}}{d \tau^{*}}
$$

which leads to

$$
\dot{\tau}-\dot{\tau}^{*}=\frac{2 \mu\left(1-2 \mathrm{~S}_{1212}\right)}{1+4 \mathrm{~S}_{1212} \mu f_{\text {Hill }}^{S}}\left(\dot{\gamma}_{p}^{*}-\dot{\gamma}_{p}\right) .
$$

The last factor in parentheses on the right hand side of Equation (39) corresponds to the difference between the applied and local plastic shear strain rates. If we consider a shell inclusion with wall volume fraction 
$f_{w}$, the plastic shear strain rate has contributions from both the plastic shearing rate within the wall $\left(\dot{\gamma}_{p}^{w}\right)$ and from the inner core $\left(\dot{\gamma}_{p}^{c}\right)$ that is proportional to their relative volume fraction, i.e.,

$$
\dot{\gamma}_{p}^{*}=f_{w} \dot{\gamma}_{p}^{w}+\left(1-f_{w}\right) \dot{\gamma}_{p}^{c}
$$

Re-arranging for $\dot{\gamma}_{p}^{c}$,

$$
\dot{\gamma}_{p}^{c}=\frac{\dot{\gamma}_{p}^{*}-f_{w} \dot{\gamma}_{p}^{w}}{\left(1-f_{w}\right)}
$$

Since the dislocation density within walls (Sauzay, 2007) is significantly higher than that from the inner core, the effective shear stress is much higher in walls. Therefore, substituting into Equation (41) and considering the plastic shearing rate of the walls as negligible gives

$$
\dot{\tau}-\dot{\tau}^{*}=\frac{2 \mu\left(1-2 \mathrm{~S}_{1212}\right)}{1+4 \mathrm{~S}_{1212} \mu f_{\text {Hill }}^{S}}\left(\dot{\gamma}_{p}^{*}-\frac{\dot{\gamma}_{p}^{*}}{\left(1-f_{w}\right)}\right) .
$$

which is rewritten as

$$
\dot{\tau}^{*}-\dot{\tau}=\dot{\tau}^{*}-\left(\dot{\tau}^{*}-\dot{B}\right)=\frac{f_{w}}{1-f_{w}} \frac{2 \mu\left(1-2 S_{1212}\right)}{1+4 S_{1212} \mu f_{\text {Hill }}^{S}} \dot{\gamma}_{p}^{*}
$$

The left hand side of Equation (43) represents the difference between the applied stress rate on the effective medium and the local stress rate in the core, which effectively corresponds to a back stress rate component, $\dot{B}$. Accordingly, by direct extension this equation can be written in terms of slip system shearing rates, yielding the back stress rate

$$
\dot{B}^{\alpha}=\frac{f_{w}}{1-f_{w}} \frac{2 \mu\left(1-2 S_{1212}\right)}{1+4 S_{1212} \mu f_{\text {Hill }}^{S}} \dot{\gamma}^{\alpha}
$$

For a prolate spheroid, the Eshelby tensor component satisfies (Mura, 1987)

$$
\begin{gathered}
S_{1212}=\frac{\pi \eta^{2}+\left(\eta^{2}-1.75-2 v_{p} \eta^{2}+2 v_{p}\right) C_{12}}{8 \pi\left(1-v_{p}\right)\left(\eta^{2}-1\right)} \\
C_{12}=\frac{2 \pi \eta\left(\eta \sqrt{\left(\eta^{2}-1\right)}-\cosh ^{-1}(\eta)\right)}{\sqrt{\left(\eta^{2}-1\right)^{3}}}
\end{gathered}
$$

in which $\eta$ was defined in Equation (8) and

$$
v_{p}=\frac{v+\frac{2}{3} \mu(1+v) f_{\text {Hill }}^{S}}{1+\frac{4}{3} \mu(1+v) f_{\text {Hill }}^{S}}
$$

The factor $\left(1-2 S_{1212}\right)$ depends on the configuration of the dislocation substructures and has a value of 0.52 for cells and 0.36 for cylinders. These values assume an orientation of the spheroid such that a screw 18 | P a g e 
dislocations satisfies the imposed kinematics. The back stress rate in Equation (44) represents the response of a homogenized quasiperiodic structure that induces residual stress in the material due to the coexistence of a plastically deformable matrix surrounded by a quasi-elastic structure of dislocation walls.

\section{Size effects}

Polycrystalline metals manifest a component of back stress that arises from the interaction among grains and does not depend on the absolute size for grain sizes above a few microns. As mentioned previously, this back stress contribution arises naturally in polycrystal plasticity by explicitly modeling individual grains. Conversely, the intragranular component of back stress depends on the size of grains or crystals. For example, Feaugas and Haddou (Feaugas and Haddou, 2003) measured the stress dependence on grain size in Ni under monotonic loading and showed that the back stress has a large dependence on grain size, often larger than that of the effective stress. Morrison and Moosbrugger (Morrison and Moosbrugger, 1997) quantified the grain size effects on back stress in Ni under cyclic loading and showed that the back stress increased much more than the friction stress for smaller grain sizes. Therefore, a significant fraction of the grain size effect on the flow stress for FCC alloys may be attributed to a dependence of the back stress on grain size.

The technical literature lacks a clear explanation for the origin of these effects, which have usually been attributed to dislocation pile-ups. However, TEM analyses of metals with medium to high SFE have not found pile-ups long enough to justify the size effects shown in experiments. Instead, analyses of dislocation configurations have shown that low energy structures are more stable than pile-ups (Kuhlmann-Wilsdorf, 2002), especially when cross slip is likely to occur. This is particularly important for dislocation structures formed under fatigue because each loading cycle provides an opportunity to disorganization pile-ups.

An alternative explanation of grain size effects relates to the relative scale of mesoscale structures compared to grains. The Eshelby inclusion model was initially proposed for an infinite crystal; however, grains are not infinite compared to the dislocation mean free path. Indeed, if the grain is about the size of the dislocation structure, $\left(d_{\text {struct }}\right.$, which depends on the applied load), the inclusion formalism is ill-defined. Thus, we argue that this scenario is equivalent to having no accommodation from the embedded medium, i.e., Kröner's inclusion with $f_{\text {Hill }}^{S}=0$; the same condition may be imposed if the spacing of integration points in a finite element scheme is nearly the same as $d_{\text {struct }}$.

In summary, in the early stages of deformation under monotonic loading, the dislocation mean free path can be comparable to the grain size, which admits a Hall-Petch type dependence. As plastic deformation progresses, dislocation structures are refined, limiting the mean free path and reducing $d_{\text {struct }}$. As dislocation structures progressively become smaller than the grain, they increase the plastic accommodation (e.g., larger number of cells). At large deformations, as shown by Feaugas and Haddou (Feaugas and Haddou, 2003), mechanical response is less sensitive to grain size. This characterization is expected to hold valid for crystals with sizes in the range about 1 to $500 \mu \mathrm{m}$. A grain size below $500 \mu \mathrm{m}$ tends to inhibit the formation of dislocation structures and grain boundaries become the dominant obstacles for dislocations (Mughrabi and Hoppel, 2010).

Thus, the ratio between mesocale structure size and grain/crystal size generally correlates with the strength of the back stress by regulating the extent of plastic accommodation under both monotonic and cyclic loading. In an infinite crystal, the degree of accommodation is described by Equation (38). In a finite crystal, accommodation is reduced (the limiting case corresponds to $d_{\text {struct }}$ being equal to the grain/crystal

size), since there is no additional accommodation from an embedding medium, i.e., $f_{\text {Hill }}^{S} \approx 0$. Although there is no direct dependence of the threshold stress (e.g., Equation (29)) on grain or crystal size, an increase in the back stress will elevate the overall stress level, which will further reduce the mesoscale structure size 
and increase the bow-out stress given in Equation (27). This indirect contribution from the grain size effect aligns well with experimental observations (Morrison and Moosbrugger, 1997).

As far as we know, the effect of finite domains on plastic accommodation has not been investigated from a theoretical standpoint. However, the effects of a finite domain size have been studied for elastic inclusions (e.g., Li et al., 2006a, 2006b), and the result is an increase in the effective shear modulus. Thus, we could expect that a finite domain increases the effective plastic shear modulus in Equation (36), which is indeed obtained by reducing $f_{\text {Hill }}^{S}$. Further investigations of the effect of finite domains on Eshelby inclusion with plastic deformation should provide a reliable pathway to inform a dependence of $f_{\text {Hill }}^{S}$ on the grain size.

\section{Model implementation for $\mathrm{Ni}$}

The present work aims to describe operative microscale and mesoscale mechanisms for various FCC metals with similar mesoscale dislocation structures. A first step towards validation requires prescribing a range for all parameters independently (i.e., from experiments and bottom-up models) and comparing the macroscopic response of single crystals with experiments. Hence, we implemented the constitutive model in finite element simulations to compare the response of pure Ni single crystals with experimental data available in the literature. This material seems ideal due to the abundance of available microscopic and macroscopic experimental data.

\section{Finite element implementation}

The framework was implemented using an ABAQUS UMAT and UEXTERNALDB subroutines (ABAQUS, 2009). Simulations employed a simple mesh containing 500 "brick" (C3D8R) elements, as shown in Figure 8. The element size is $50 \mu \mathrm{m}$, which is much larger than the typical spacing between walls. Single crystal meshes are oriented such that the loading direction lies along the following crystal directions: [001],[011],[111], and [149]. The first three single crystal orientations correspond to the vertices of the inverse pole figure and the latter to a single slip case. For polycrystal simulations, random orientation distributions of grains are assigned for simplicity for purposes of demonstrating collective response. Each element represents a single grain, as shown in Figure 8; the effects of higher degree of mesh refinement to represent microstructure is discussed in Appendix A. Displacements (strain) is specified along the X axis, along which periodic boundary conditions are imposed. The remaining four faces of the mesh are not constrained and represent traction free surfaces. 


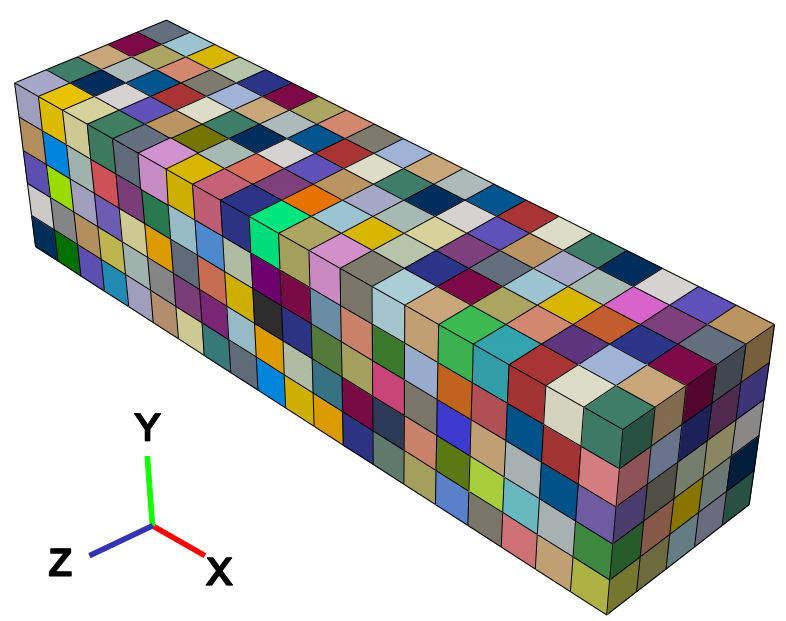

Figure 8. Example voxellated finite element mesh with 500 elements and uniaxial periodic boundary conditions. Colors correspond to different crystallographic orientations for polycrystals.

\section{Decoupling time scales}

Computational approaches that attempt to reproduce physically-based mechanisms have an intrinsic limitation when dealing with cyclic loading: Cyclic deformation evolves microstructure over thousands or millions of physical cycles in the high cycle fatigue regime. Since it is currently computationally infeasible or impractical to perform crystal plasticity calculations undergoing millions of computational cycles, an acceleration procedure should be implemented, especially for engineering applications. Castelluccio and McDowell (Castelluccio and McDowell, 2014) have previously introduced the distinction between physical and computation cycles to model early fatigue crack growth meandering through a set of grains.

Decoupling time scales under cyclic loading has been an area of active research and different strategies have been proposed. For example, Ghosh and coworkers (Ghosh and Chakraborty, 2013) have proposed a computational approach based on wavelet transformation method to achieve accelerated time scaling of evolving fluctuating mechanical fields. For the present work, we appeal to established concepts regarding the relative rates at which cyclic plasticity and microstructure evolve with respect to accumulated cycles. After a few hundred cycles in low cycle fatigue or a few thousand loading cycles for transition fatigue and beyond, stable dislocation structures typically emerge in smooth specimens, resulting in stabilized shape loop factor and peak stress (Morrison and Aubin, 1994; Polak et al., 1988). Indeed, minor changes on macroscopic stress may occur over thousands of cycles, and many times these changes correspond to the formation of new saturation structures at other, almost pristine locations. Therefore, we argue that transient development of mesoscale dislocation substructures are responsible for the initial evolution. We can impose the expected structures under saturation, and evolve towards them in a computationally accelerated manner.

The competition between the computational burden and modeling of realistic mechanisms is therefore addressed here by decoupling the time scales of imposed cyclic loading and microstructure evolution by accelerating the latter towards cyclically stable conditions. This decoupling strategy enforces that mesoscale structures reach their saturation condition much faster in terms of computational cycles than in actual experimentally cycling, thereby obviating the need to apply thousands of computational cycles. The following assumptions are made:

- Mesoscale dislocation structures evolve only on a cycle-by-cycle basis according to phenomenological strain-based models calibrated to the saturation state. The selection of structures shown in Figure 4 corresponds to the set of possible saturation states, and these structures are updated once per full computational cycle. 
- Dislocation density evolves continuously during each loading cycle to accommodate imposed cyclic deformation.

This methodology rapidly achieves the saturated state over a small number of computational cycles, but it can also introduce numerical instabilities if dislocation structure variables are updated instantaneously. To avoid such instabilities and improve convergence, numerical viscosity was implemented in the structural parameters $\eta_{\text {struct }}, d_{\text {struct }}$ and $f_{w}$ such that they are updated over a few hundred time increments at the beginning of each cycle. Furthermore, the value of the structure parameters transferred to finite element calculations corresponds to the average over the last four full cycle estimates; this average smooths out substructure evolution and accelerates saturation of the overall cyclic stress-strain response, typically achieving this condition after five to ten computational cycles.

A secondary effect of this acceleration strategy is that the back stress may rise steeply during one loading cycle, which results in asymmetry of the stress-strain hysteresis under completely reversed straining. This behavior does not occur if the parameters evolve gradually during tension and compression. On the contrary, we can expect such asymmetry due to overloads. To mitigate these effects under completely reversed cyclic loading, we subtract the mean back stress per cycle from the back stress at the beginning of each cycle. This simplification implies the mean average accumulation of dislocations do not have a net sign for completely reversed loading. As a result, the appropriate completely reversed back stress amplitude is achieved at saturation. Different strategies are envisioned for cyclic loading with high mean stress such as progressive increase of the peak applied stress. We emphasize that our simplifications will not impact other loading conditions as long as dislocation structures do not develop a significant net sign.

Finally, we emphasize that the constitutive framework is physically-based and robust, so that if computational resources are available, cycle-by-cycle evolution of dislocation substructure and stress-strain response could be tracked. Its rate of evolution would correspond to the progression of the net dislocation activity over each cycle and can be adjusted as necessary to accommodate experimental observations.

\section{Material parameters and physical constants}

The crystal plasticity framework depends on three types of parameters: Direct, stochastic and initial parameters. Direct parameters correspond to physical constants associated with the lattice and lattice defect interactions, and do not depend on the mesoscopic response of a statistical ensemble. These parameters are deduced for pure $\mathrm{Ni}$ from experimental measurements or bottom-up calculations and are linked to fundamental physics. These include:

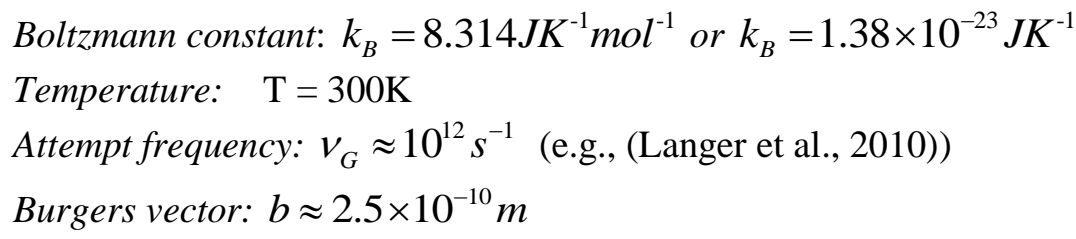

The elastic constants are also considered as direct quantities that can be computed using bottom-up approaches (even when they are the result of a quantum statistical ensemble). We employ the extensive and detailed experimental results from Ledbetter (Ledbetter, 1973) that assume cubic symmetry. Furthermore, the shear modulus is computed based on the Hershey polycrystal average, i.e.,

$$
\begin{aligned}
& \text { Elastic constants @ 300K (Ledbetter, 1973): } \\
& C_{11}=249 \mathrm{GPa} \\
& C_{12}=155 \mathrm{GPa} \\
& C_{44}=114 \mathrm{GPa}
\end{aligned}
$$




$$
\begin{aligned}
& C_{44} \text { at } 0 K=127.6 \mathrm{GPa} \\
& \text { Shear modulus at } 300 \mathrm{~K}: \mu=80.6 \mathrm{GPa}
\end{aligned}
$$

Stochastic parameters are associated with mechanisms or theoretical models that describe the behavior of a statistical ensemble. Among these quantities are energy barrier parameters, dislocation interaction strengths, the cross slip activation energy and volume, etc. These quantities can be estimated by indirect experimental measurements and with advisement of simulations such as dislocation dynamics. Stochastic parameters typically have much larger uncertainty than direct parameters.

The parametrization of the dislocation energy barrier can be estimated with atomistic simulations (Narayanan et al., 2014), but this requires a dominance of a specific deformation mechanism (e.g., dislocation cross slip or obstacle by-pass). An independent approach is offered by calibration of these parameters by comparing models with the early deformation of single crystals under monotonic loading. This strategy relies on the fact that the dominant energy barrier parametrization at the initial yield point does not depend strongly on hardening mechanisms, which are relatively inactive in the early deformation of well annealed metals with low dislocation densities.

Hence, we implemented a monotonic simulation to estimate the parameters by comparing the early yield point with experiments, as shown in Figure 9. The constitutive model for monotonic loading is the similar to that described except that $\eta_{\text {struct }} \approx 1, K_{\text {struct }} \approx 12$, and the wall thickness evolution from Estrin et al. (Estrin et al., 1998); these correspond to a rough scaling of dislocation substructures under monotonic loading. The activation energy and profile parameters were adjusted to match the yield stress and the span of Stage I monotonic deformation with bounds $0<p<1$ and $1<q<2$. An adequate match of the data is obtained for $p=0.9, q=1.5, F_{0}=0.985 \mathrm{ev}$, and $s_{t}^{0}=100 \mathrm{MPa}$, but we note that they are not unique for reproducing the experimental response. These values corresponds to weak obstacle strength (Frost and Ashby, 1982), which goes along with the hypothesis that most plastic deformation comes from dislocation production in between walls. In addition, these parameters should not depend on the loading conditions, but vary among different materials.
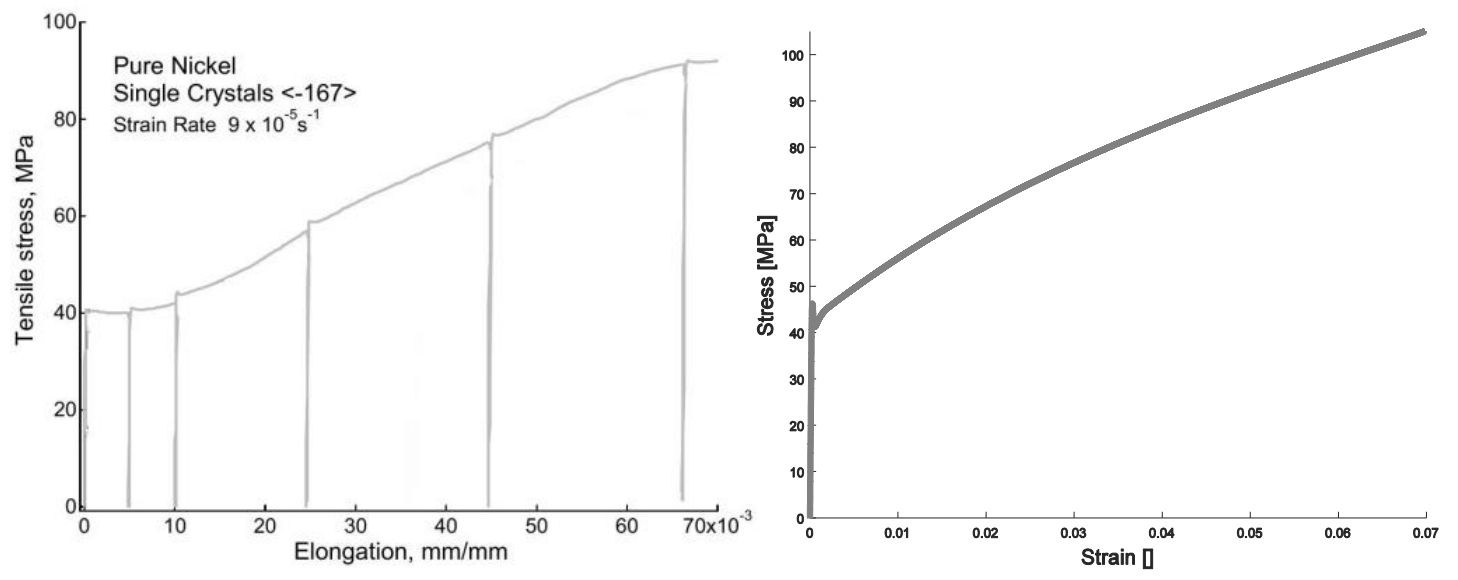

Figure 9. Monotonic tensile behavior of Ni single crystal oriented for uniaxial loading along the [167] orientation: (left) experiments from Yagodzinskyy et al., 2008, and (right) simulation.

Dislocation interaction strengths may be estimated by measuring dislocation curvature and spacing in TEM micrographs or from dislocation dynamics simulations. Parameters are estimated as:

Annihilation distance for edge dislocations (not induced by cross slip): $y_{e} \approx 3 \mathrm{~nm}$ (Aslanides and Pontikis, 1998)

23 | P a g e 
Annihilation distance for screw dislocations (induced by cross slip): $y_{s} \approx 13 \mathrm{~nm}$ (Feaugas, 1999)

Intrinsic lattice friction: $S_{0}^{\alpha} \approx 4 \mathrm{MPa}$

Dislocation-dislocation self-interaction: $A_{i i} \approx 0.1$ (Various authors have proposed a range of values, cf. (Kubin et al., 2008; Fivel et al., 1998; Gerard et al., 2013; Kubin et al., 2008; Schwartz et al., 2013).

Line tension coefficient: $\alpha_{L E} \approx 1$

Cross slip activation volume: $V_{C S}^{0} \approx 1000 b^{3}$ to reproduce activation volumes close to those in (Armstrong and Rodriguez, 2006).

Cross slip efficiency: $\phi_{C S} \approx 0.5$

We consider mesoscale length scales that control work hardening and back stresses. These parameters represent a mean value for specific dislocation structures: $\eta_{\text {cell }} \approx 1, \eta_{\text {labyr }} \approx 2, \eta_{P S B} \approx 20, \eta_{\text {veins }} \approx 50$. Furthermore, $K_{\text {struct }} \approx 3.75$ based on Bretschneider et al., 1997 and Sauzay and Kubin, 2011, while $k_{\text {multi }}=2$ for all structures (Essmann and Mughrabi, 1979), except cells for which $k_{\text {multi }}^{\text {Cell }}=1$.

The parameters in Equation (9) have a large uncertainty and their experimental quantification requires measuring dislocation structures in single crystals as a function of applied strain. Furthermore, the calculation of $f_{w}$ assumes a geometrical configuration of walls that is subject to various assumptions or idealizations. For instance, Estrin et al., 1998; Feaugas and Gaudin, 2004; Nahm et al., 1977; Toth et al., 2002 proposed four different geometric models for computing $f_{w}$ that depend on wall thickness and spacing measurements.

Here we have approximated the parameters in Equation (9) as $f_{\text {inf }}=0.24, f_{0}=0.4, g_{p}=1.7$. Figure 10 plots the wall fraction parametrization and the experimental data available for different materials and loading conditions from Tippelt et al. (Tippelt et al., 1997) and Ahmed et al. (Ahmed et al., 2006). The error bars represent the variability the calculations for the four geometric models mentioned before. Evidently, the wall fraction should be regarded as a simple and approximate internal state variable that depends on the geometry of dislocation structures. 


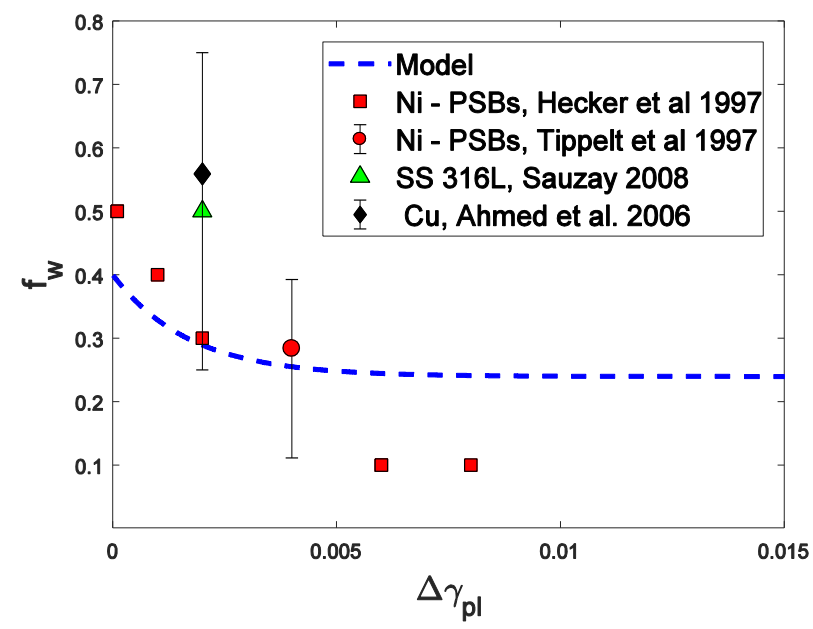

Figure 10. Dependence of the wall fraction, $f_{w}$, as a function of the maximum crystallographic plastic shear range at saturation. Data points and error bars corresponds to quantities computed from experimental data (Ahmed et al., 2006; Hecker et al., 1997; Sauzay, 2008; Tippelt et al., 1997).

Finally, internal state variables need to be initialized. Here is a summary of the initial values implemented:

Initial Density of mobile (screw) dislocations: $\rho_{m 0}^{s}=10^{10} \mathrm{~m}^{-2}$

The initial structural distance is $d_{\text {struct }}^{0}=\frac{1}{\sqrt{\rho_{m 0}^{s}}}=10 \mu \mathrm{m}$

The normalized glide $\eta=\eta_{\text {veins }}$ The wall fraction is initialized at relatively low values to improve convergence $f_{w}^{0}=0.25$

The cross slip activation volume is $V_{C S}=1000 b^{3}$, for maximum shear stress below 5 MPa.

\section{Model Results}

Figure 11 presents the cyclic stress-strain curve (mean peak stress and strain) from simulations and experiments for orientation of loading for four Ni single crystals: [149], [001], [011], and [111] for completely reversed straining $\left(\mathrm{R}_{\varepsilon}=-1\right)$ at a strain rate of $0.001 \mathrm{~s}^{-1}$. Simulations and experiments agree in terms of their relative stress levels and general trends (note the large uncertainty for similar tests from different authors). Accurate prediction of the peak stresses and strains are of primary interest since these quantities usually inform fatigue predictions (Castelluccio et al., 2014). Prediction of the entire hysteresis loop is a major challenge, and it may not provide much more fidelity to fatigue predictions. Instead, the area under the cyclic loop, which carries information about the entire loop, may be useful in the prediction of fatigue behavior. 


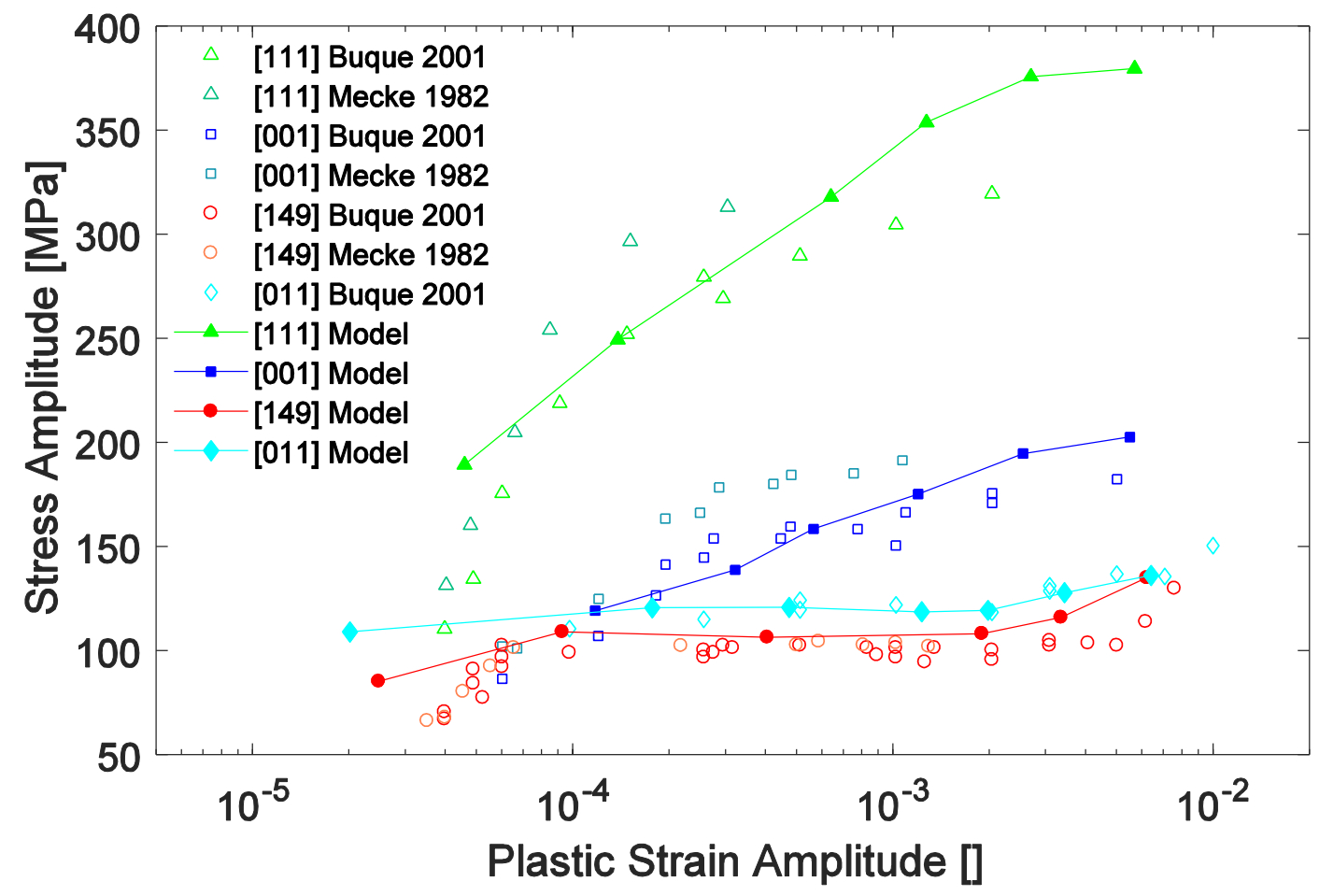

Figure 11. Cyclic stress-strain curve comparison among models and experiments (Buque, 2001; Mecke and Blochwitz, 1982) for four pure Ni single crystals under completely reversed strain-controlled uniaxial loading $\left(\mathrm{R}_{\varepsilon}=-1\right)$ along [001], [011], [111], [149] directions.

Figure 12 presents the evolution of the cyclic peak stress over 16 computational cycles for various single crystals subjected to $0.25 \%$ strain amplitude for $\mathrm{R}_{\varepsilon}=-1$. After initial oscillation, the time scale decoupling effectively achieves saturated cyclic peak stress. 


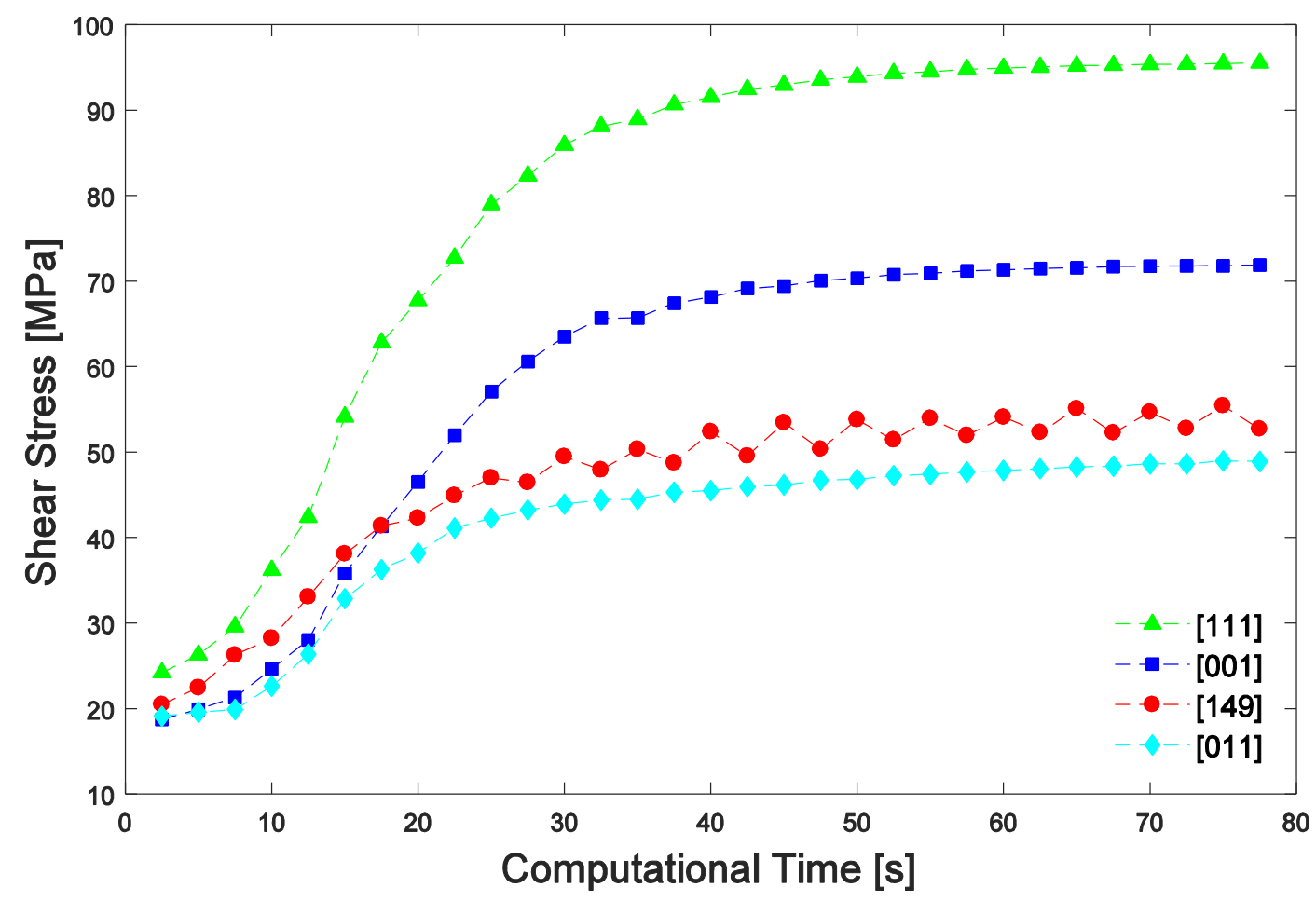

Figure 12. Convergence of the cyclic peak stress with computational cycles under $0.25 \%, R_{\varepsilon}=-1$ for four pure Ni single crystals under uniaxial loading along [001], [011], [111], [149] directions.

Figure 13 presents the saturated uniaxial cyclic stress-strain loops from simulations at various strain amplitudes for Ni single crystals loaded uniaxially along the [001], [011], [111], and [149] directions. By comparing the yield and ultimate stress, we observe that the back stress has a larger impact for cells and labyrinth structures ([001] and [111] crystals). Some of these results present sharper transition between elastic and plastic behavior than is usually observed in macroscopic tests. We attribute such a difference due to specimen size effects (e.g., constraint gradients) and variability in dislocation structures (e.g., the standard deviation in Equation(16)). Experimental research in response of nanopillars has exhibited this kind of sharp transition from elastic to plastic deformation. 

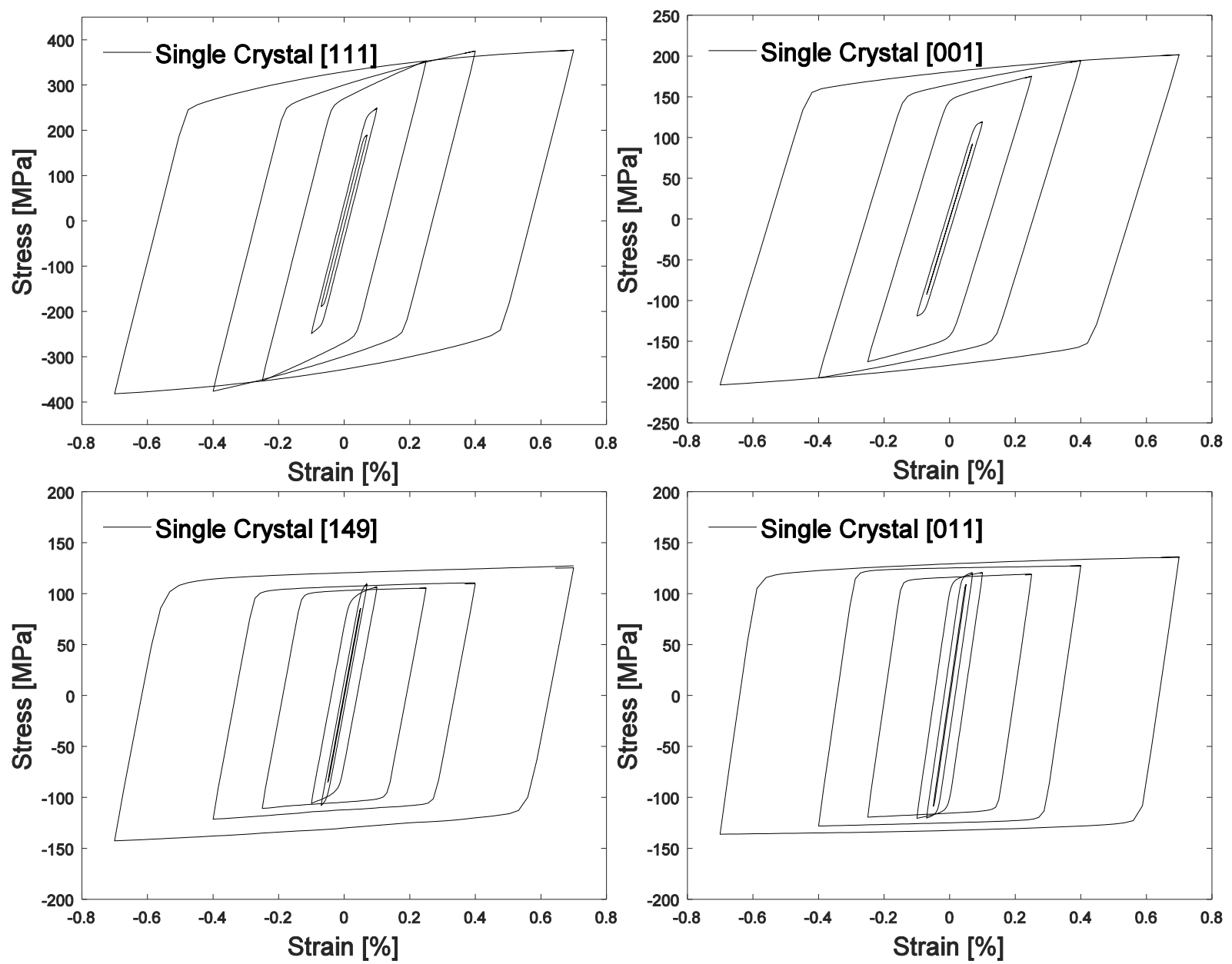

Figure 13. Uniaxial stress-strain curves for different Ni single crystals loaded along [111], [001], [149], [011] directions.

Figure 14 presents the misorientation distribution that results from the mesoscale perturbation of the Euler angles in Equation (15). Clearly, the distribution based on stochastic dynamics is able to reproduce misorientation distributions that resemble experimental results (Figure 6). All parameters in Equation (15) arise from theoretical arguments except for $K_{M C D} \approx 0.2$, whose value approximately corresponds to the most likely misorientation upon $1 \%$ cyclic deformation. 


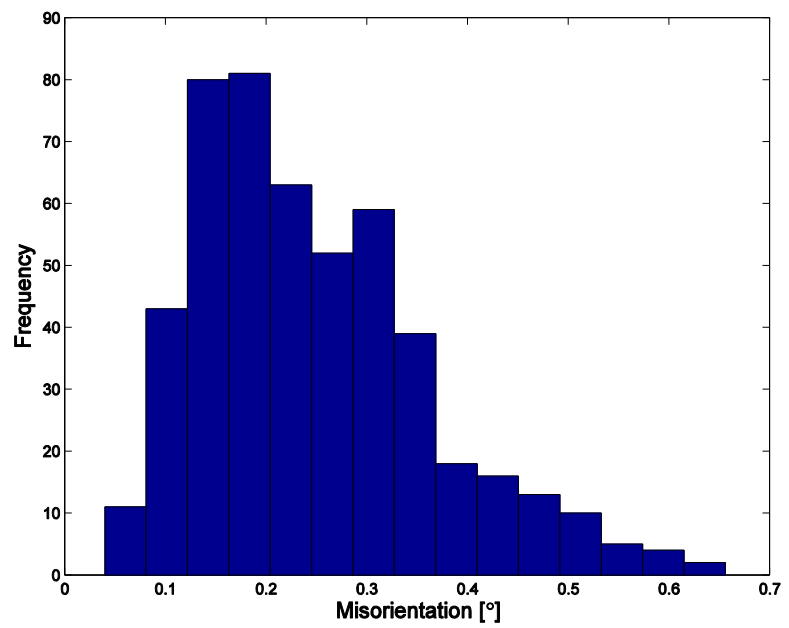

Figure 14. Frequency distribution of grain misorientation for $0.7 \%$ cyclic deformation computed from simulations of Ni single crystals oriented along [111].

Finally, we seek to extend the validation of the constitutive framework by comparing cyclic stressstrain hysteresis loops from polycrystalline experiments and models. Figure 15 compares the experimental data (symbols) for coarse $(290 \mu \mathrm{m})$ and fine $(24 \mu \mathrm{m})$ grain sizes from Morrison and Moosbrugger, 1997 with simulations (lines) with no accommodation ( $f_{\text {hill }}=0$, corresponding to a very fine grain size) and full accommodation (Equation (38), for a seemingly infinite grains or crystals, in practical terms larger than $1 \mathrm{~mm}$ ). Similarly, we compare the cyclic stress-strain curves (mean peak stress and strain) from simulations and experiments for polycrystalline specimens Figure 16. Clearly, both computational results bound the experimental results in both figures. Future efforts should attempt to clarify the role of grain/crystal size on the accommodation factors and other size effects such as grain boundary slip transmission to match the experiments with less than $10 \%$ error. 

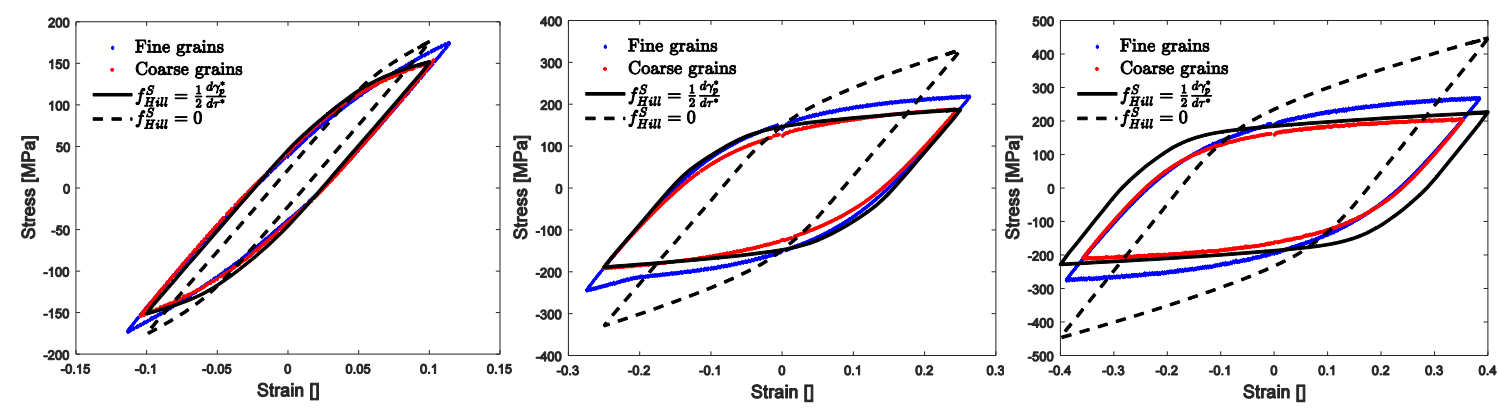

Figure 15. Comparison of uniaxial stress-strain responses for polycrystalline Ni specimens from experiments and simulations. Symbols correspond to experimental data for coarse and fine grain sizes

(Morrison and Moosbrugger, 1997). Lines correspond to two model extremes $f_{\text {Hill }}^{S}=0$ (no

$$
\text { accommodation) and } f_{H i l l}^{S}=\frac{1}{2} \frac{d \gamma_{p}^{*}}{d \tau^{*}}(\text { Equation (38)). }
$$

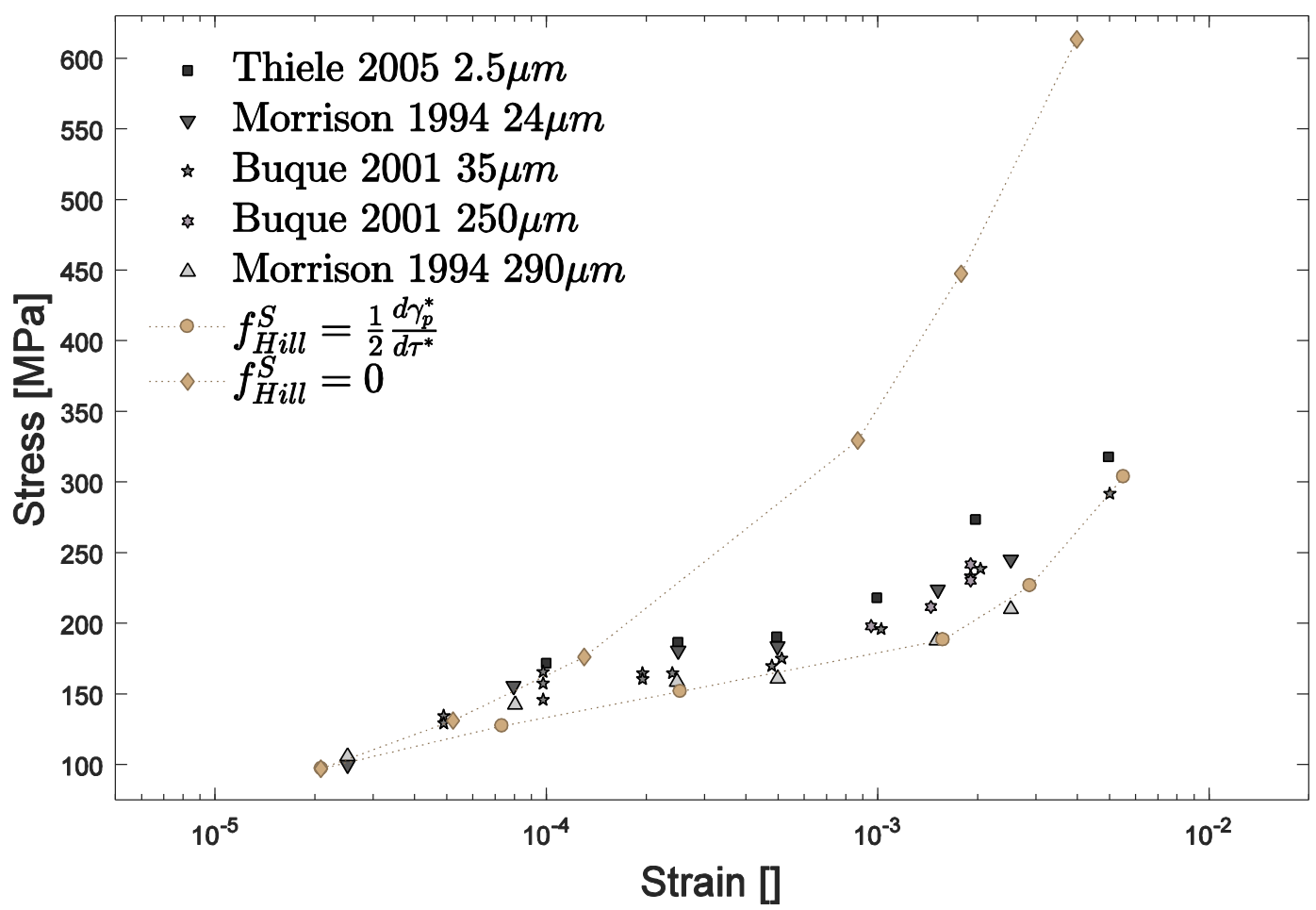

Figure 16. Cyclic stress-strain curve comparison between experiments and models for pure $\mathrm{Ni}$ polycrystalline specimens under uniaxial, completely reversed strain-controlled loading $\left(R_{\varepsilon}=-1\right)$. Rhomboid dotted symbol corresponds to $f_{\text {Hill }}^{S}=0$ (no accommodation) and circle dotted symbol to $f_{\text {Hill }}^{S}=\frac{1}{2} \frac{d \gamma_{p}^{*}}{d \tau^{*}}$ (Equation (38)). Other symbols correspond to experimental data for different grain sizes from Buque et al., 2001; Morrison, 1994; Thiele et al., 2005. 


\section{Discussion}

This research is motivated by the demanding challenge to develop a constitutive model that is capable of reproducing the cyclic behavior of FCC single crystals and polycrystals based only on parameters that can be directly measured with experiments or quantified with bottom-up models. Pursuit of this objective requires revising some of the simplifications commonly employed in constitutive models. We introduced the notion that the mean free path is determined by mesoscale dislocation structures rather than dislocation densities. This divergence from traditional models introduces plastic anisotropy that depends on the structure and crystal orientation. For example, a cell structure requires an order of magnitude larger dislocation density than PSBs to provide identical crystallographic shear.

A second important contribution of this paper is the derivation of a physically-based back stress that depends on the mesoscale dislocation structures. This approach allows to predict rather than fit the back stress by informing the model with mesoscale information from TEM, dislocation dynamics etc. Furthermore, it provides the basis to estimate and compare the different back stress induced in different metallic materials and size effects. Similarly, the distinction of annihilation distances that result from cross slip processes (e.g., edge vs screw dislocation annihilation) induces further anisotropy among crystallographic orientations.

Another important notion introduced in this work is the distinction of parameters that can be measured directly and those that represent the mean state of a many-body problem. At the mesoscale, elastic constants have a much lower uncertainty than dislocation wall thickness or annihilation distances. Furthermore, our approach to accelerate computational cycling to achieve cyclically stable substructures limits computational effort. This work focuses on describing the saturated dislocation substructures under completely reversed loading, but the proposed framework should also apply to transients.

There are various aspects that require further investigation. For example, the mechanisms that are responsible for the development of dislocation structures are not fully understood and the assumption that the mean free paths follow similitude for all structures. Future work in discrete dislocation dynamics and continuum modeling should explore approaches to describe the formation and transient evolution of dislocation walls to inform constitutive relations of the type discussed here. Another aspect that requires further investigation is the extent of plastic deformation of dislocation walls. Our approach considered elastic walls that refine with increasing plastic deformation, but wall thickness may saturate at high strains and walls may deform plastically; this might be particularly important for monotonic large deformation cases. Further research is also required to understand size effects. Both single and polycrystal behavior depends on the size of the specimens and grains, which has only been considered by analyzing extreme cases and assuming single crystals are much larger than characteristic mesoscale structures.

\section{Conclusions}

This work has introduced a crystal plasticity framework that emphasizes the role of mesoscale dislocation structures such as cells and PSBs in cyclic stress-strain behavior. We have focused on reproducing both single- and polycrystal response under saturated cyclic loading conditions in an effort to link macroscopic and microscopic phenomena. The assessment of single crystal behavior is of primary importance to enhance the prediction of plastic deformation activity among slip systems.

We introduced a back stress formulation that is directly related to mesoscale structures by means of the Eshelby's inclusion and Hill's constraint tensor. In addition, we proposed a scheme to predict the most likely dislocation structure, which is validated for four single crystal orientations

The modeling approach showed enough flexibility to reproduce the macroscopic response while following physically-based principles under various loading amplitudes and crystal orientations. Although we implemented the calculations for pure $\mathrm{Ni}$, the framework is general for FCC crystals with medium to

31 I P a g e 
high SFE. Future efforts will focus on unified approaches valid for monotonic and cyclic loading of single crystals and polycrystals in other FCC metals such as copper and austenitic stainless steels.

\section{Acknowledgments}

The authors are grateful for the support of QuesTek Innovations LLC under contract from the Naval Nuclear Laboratory, operated by Bechtel Marine Propulsion Inc. for the US Naval Reactors Program, with Dr. Clint Geller as research coordinator. DLM also appreciates the support of the Carter N. Paden, Jr. Distinguished Chair in Metals Processing at Georgia Tech.

\section{Appendix A}

The representation of grains in Figure 8 assumes one element per grain, which is very coarse and does not convey a high fidelity representation of the microstructure. Nevertheless, Sauzay et al., 2014 showed that the coarse representation of grains does not substantially affect the simulated macroscopic response nor the cumulative probability distribution of the mean plastic strain per grain. Similarly, the present authors (Castelluccio and McDowell, 2015) show equivalent distributions of fatigue indicator parameters at the grain level among various microstructural refinements. Certainly, refined meshes are required to assess local damage at crack tips or grain boundaries.

Since the assessment of cyclic stress-strain curves in Figure 16 requires many time-consuming simulations, we opted to employ the simplified meshes in Figure 8. To estimate the effects of a refined mesh we performed an identical simulation with 4000 elements and 283 equiaxed grains, as shown in Figure 17. The results in Figure 18 show a close agreement of the overall stress-strain behavior between the coarse and refined mesh representations of grains, the most notable difference being a slight increase in the back stress for the refined mesh. These results reaffirm that coarse meshes are sufficient for assessing overall stress-strain behavior and suggest a minor dependence of the intergranular back stress on the details of the grain morphology.

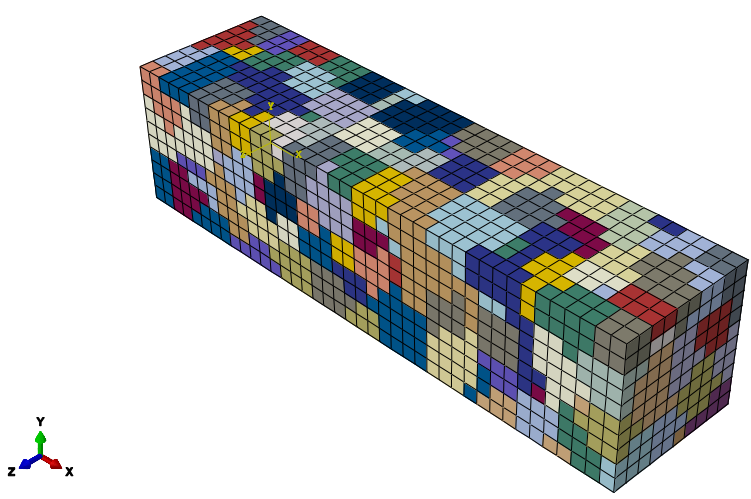

Figure 17. Example voxellated refined finite element mesh with 4000 elements, 283 equiaxed grains and uniaxial periodic boundary conditions. 


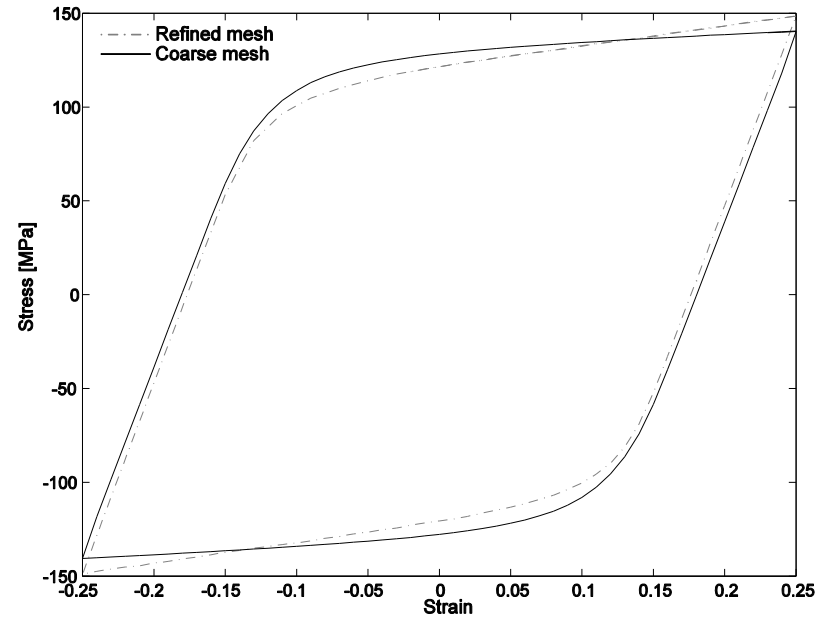

Figure 18. Comparison of uniaxial stress-strain responses from polycrystalline Ni models with coarse and fine meshes.

\section{References}

ABAQUS, 2009. FEM software V6.9, Simulia Corp., Providence, RI, USA. Simulia, Inc., Providence, RI, USA. Ahmed, J., Roberts, S.G., Wilkinson, A.J., 2006. Characterizing dislocation structure evolution during cyclic deformation using electron channelling contrast imaging. Philos. Mag. 86, 4965-4981. doi:10.1080/14786430600710941

Ananthakrishna, G., 2007. Current theoretical approaches to collective behavior of dislocations. Phys. Rep.-Rev. Sect. Phys. Lett. 440, 113-259. doi:10.1016/j.physrep.2006.10.003

Argon, A.S. (Ed.), 1975. Constitutive Equations in Plasticity, 1St Edition edition. ed. The MIT Press, Cambridge, Mass.

Armstrong, R.W., Rodriguez, P., 2006. Flow stress/strain rate/grain size coupling for fcc nanopolycrystals. Philos. Mag. 86, 5787-5796. doi:10.1080/14786430600764872

Arsenlis, A., Parks, D.M., 2002. Modeling the evolution of crystallographic dislocation density in crystal plasticity. J. Mech. Phys. Solids 50, 1979-2009. doi:10.1016/S0022-5096(01)00134-X

Asaro, R. J., 1983. Crystal plasticity. J. Appl. Mech. 50, 921-934.

Asaro, Robert J., 1983. Micromechanics of Crystals and Polycrystals, in: John W. Hutchinson and Theodore Y. Wu (Ed.), Advances in Applied Mechanics. Elsevier, pp. 1-115.

Asaro, R.J., Needleman, A., 1985. Overview 42. Texture development and strain-hardening in rate dependent polycrystals. Acta Metall. 33, 923-953.

Aslanides, A., Pontikis, V., 2000. Numerical study of the athermal annihilation of edge-dislocation dipoles. Philos. Mag. A 80, 2337-2353. doi:10.1080/01418610008216476

Aslanides, A., Pontikis, V., 1998. Atomistic study of dislocation cores in aluminium and copper. Comput. Mater. Sci. 10, 401-405.

Berveiller, M., Zaoui, A., 1979. An extension of the self-consistent scheme to plastically-flowing polycrystals. J. Mech. Phys. Solids 26, 325-344. doi:10.1016/0022-5096(78)90003-0

Blochwitz, C., Veit, U., 1982. Plateau Behaviour of Fatigued FCC Single Crystals. Cryst. Res. Technol. 17, 529-551. doi:10.1002/crat.2170170502

Bonneville, J., Escaig, B., Martin, J.L., 1988. A study of cross-slip activation parameters in pure copper. Acta Metall. 36, 1989-2002. doi:10.1016/0001-6160(88)90301-X 
Bretschneider, J., Holste, C., Tippelt, B., 1997. Cyclic plasticity of nickel single crystals at elevated temperatures. Acta Mater. 45, 3775-3783. doi:10.1016/S1359-6454(97)00030-X

Brown, L.M., 2002. A dipole model for the cross-slip of screw dislocations in FCC metals. Philos. Mag. A 82, 1691-1711. doi:10.1080/01418610208235684

Buque, C., 2001. Dislocation structures and cyclic behaviour of [011] and [111]-oriented nickel single crystals. Int. J. Fatigue 23, 671-678. doi:10.1016/S0142-1123(01)00032-9

Buque, C., Bretschneider, J., Schwab, A., Holste, C., 2001. Dislocation structures in cyclically deformed nickel polycrystals. Mater. Sci. Eng. A 300, 254-262. doi:10.1016/S0921-5093(00)01784-6

Castelluccio, G.M., McDowell, D.L., 2015. Microstructure and mesh sensitivities of mesoscale surrogate driving force measures for transgranular fatigue cracks in polycrystals. Mater. Sci. Eng. A 639, 626-639. doi:10.1016/j.msea.2015.05.048

Castelluccio, G.M., McDowell, D.L., 2014. Mesoscale modeling of microstructurally small fatigue cracks in metallic polycrystals. Mater. Sci. Eng. A 598, 34-55. doi:10.1016/j.msea.2014.01.015

Castelluccio, G.M., Musinski, W.D., McDowell, D.L., 2014. Recent developments in assessing microstructure-sensitive early stage fatigue of polycrystals. Curr. Opin. Solid State Mater. Sci. 18, 180-187. doi:10.1016/j.cossms.2014.03.001

Cheng, A.S., Laird, C., 1981. Mechanisms of fatigue hardening in copper single crystals: The effects of strain amplitude and orientation. Mater. Sci. Eng. 51, 111-121. doi:10.1016/00255416(81)90112-9

Cleri, F., 2005. Evolution of dislocation cell structures in plastically deformed metals. Comput. Phys. Commun., Proceedings of the Europhysics Conference on Computational Physics 2004 CCP 2004 Europhysics Conference on Computational Physics 2004 169, 44-49. doi:10.1016/j.cpc.2005.03.012

Conti, S., Ortiz, M., 2005. Dislocation Microstructures and the Effective Behavior of Single Crystals. Arch. Ration. Mech. Anal. 176, 103-147.

Cottrell, A.H., 1953. Dislocations and Plastic Flow in Crystals. Clarendon P.

El-Awady, J.A., Ghoniem, N.M., Mughrabi, H., 2007. Dislocation modelling of localized plasticity in persistent slip bands. Presented at the TMS 2007, The Minerals, Metals \& Materials Society, pp. 23-35.

Eshelby, J.D., 1957. The Determination of the Elastic Field of an Ellipsoidal Inclusion, and Related Problems. Proc. R. Soc. Lond. Ser. Math. Phys. Sci. 241, 376-396. doi:10.1098/rspa.1957.0133

Essmann, U., Mughrabi, H., 1979. Annihilation of dislocations during tensile and cyclic deformation and limits of dislocation densities. Philos. Mag. A 40, 731-756. doi:10.1080/01418617908234871

Estrin, Y., Brechet, Y., Braasch, H., 1996. A Dislocation Density Based Constitutive Model for Cyclic Deformation. J. Eng. Mater. Technol. 118, 441-447. doi:10.1115/1.2805940

Estrin, Y., Tóth, L.S., Molinari, A., Bréchet, Y., 1998. A dislocation-based model for all hardening stages in large strain deformation. Acta Mater. 46, 5509-5522. doi:10.1016/S1359-6454(98)00196-7

Feaugas, X., 1999. On the origin of the tensile flow stress in the stainless steel AISI 316L at $300 \mathrm{~K}$ : Back stress and effective stress. Acta Mater. 47, 3617-3632.

Feaugas, X., Gaudin, C., 2004. Ratchetting process in the stainless steel AISI 316L at $300 \mathrm{~K}$ : an experimental investigation. Int. J. Plast. 20, 643-662. doi:10.1016/S0749-6419(03)00076-7

Feaugas, X., Haddou, H., 2003. Grain-size effects on tensile behavior of nickel and AISI 316L stainless steel. Metall. Mater. Trans. A 34, 2329-2340. doi:10.1007/s11661-003-0296-5

Fivel, M., Tabourot, L., Rauch, E., Canova, G., 1998. Identification through mesoscopic simulations of macroscopic parameters of physically based constitutive equations for the plastic behaviour of fcc single crystats. J. Phys. IV 08, Pr8-151-Pr8-158. doi:10.1051/jp4:1998819

34 | P a g e 
Frederick, C.O., Armstrong, P.J., 2007. A mathematical representation of the multiaxial Bauschinger effect. Mater. High Temp. 24, 1-26.

Frost, H.J., Ashby, M.F., 1982. Deformation-mechanism maps: the plasticity and creep of metals and ceramics. Pergamon Press, Oxford [Oxfordshire]; New York.

Gerard, C., Cailletaud, G., Bacroix, B., 2013. Modeling of latent hardening produced by complex loading paths in FCC alloys. Int. J. Plast. 42, 194-212. doi:10.1016/j.ijplas.2012.10.010

Ghosh, S., Chakraborty, P., 2013. Microstructure and load sensitive fatigue crack nucleation in Ti-6242 using accelerated crystal plasticity FEM simulations. Int. J. Fatigue 48, 231-246. doi:10.1016/j.ijfatigue.2012.10.022

Hansen, B.L., Bronkhorst, C.A., Ortiz, M., 2010. Dislocation subgrain structures and modeling the plastic hardening of metallic single crystals. Model. Simul. Mater. Sci. Eng. 18, 005500 1-42.

Hecker, M., Thiele, E., Holste, C., 1997. X-ray diffraction analysis of internal stresses in the dislocation structure of cyclically deformed nickel single crystals. Mater. Sci. Eng. A 234-236, 806-809. doi:10.1016/S0921-5093(97)00370-5

Hennessey, C., Castelluccio, G.M., McDowell, D.L., 2017. Sensitivity of polycrystal plasticity to slip system kinematic hardening laws for Al 7075-T6. Mater. Sci. Eng. A 687, 241-248. doi:10.1016/j.msea.2017.01.070

Hill, R., 1965. Continuum micro-mechanics of elastoplastic polycrystals. J. Mech. Phys. Solids 13, 89-101. doi:10.1016/0022-5096(65)90023-2

Hughes, D.A., Chrzan, D.C., Liu, Q., Hansen, N., 1998. Scaling of Misorientation Angle Distributions. Phys. Rev. Lett. 81, 4664-4667. doi:10.1103/PhysRevLett.81.4664

Hughes, D.A., Liu, Q., Chrzan, D.C., Hansen, N., 1997. Scaling of microstructural parameters: Misorientations of deformation induced boundaries. Acta Mater. 45, 105-112. doi:10.1016/S1359-6454(96)00153-X

Kamaya, M., Kuroda, M., 2011. Fatigue Damage Evaluation Using Electron Backscatter Diffraction. Mater. Trans. 52, 1168-1176. doi:10.2320/matertrans.M2011014

Khan, A., Huang, S., 1995. Continuum theory of plasticity. Wiley, New York.

Kocks, U.F., Argon, A., Ashby, M.F., 1975. Thermodynamics and kinetics of slip. Prog. Mater. Sci. 19.

Kocks, U.F., Mecking, H., 2003. Physics and phenomenology of strain hardening: the FCC case. Prog. Mater. Sci. 48, 171-273. doi:doi: DOI: 10.1016/S0079-6425(02)00003-8

Kröner, E., 1961. Zur plastischen verformung des vielkristalls. Acta Metall. 9, 155-161. doi:10.1016/0001-6160(61)90060-8

Kubin, L., Devincre, B., Hoc, T., 2008. Modeling dislocation storage rates and mean free paths in facecentered cubic crystals. Acta Mater. 56, 6040-6049. doi:10.1016/j.actamat.2008.08.012

Kubin, L.P., Canova, G., Condat, M., Devincre, B., Pontikis, V., Bréchet, Y., 1992. Dislocation Microstructures and Plastic Flow: A 3D Simulation. Solid State Phenom. 23-24, 455-472. doi:10.4028/www.scientific.net/SSP.23-24.455

Kuhlmann-Wilsdorf, D., 2002. Chapter 59 The LES theory of solid plasticity, in: F.R.N. Nabarro and M.S. Duesbery (Ed.), Dislocations in Solids. Elsevier, pp. 211-342.

Kuhlmann-Wilsdorf, D., 1962. A new theory of work hardening in crystals. Trans. Met. Soc. AIME 218, 962.

Langer, J.S., 2015. Statistical thermodynamics of strain hardening in polycrystalline solids. Phys. Rev. E 92, 032125. doi:10.1103/PhysRevE.92.032125

Langer, J.S., Bouchbinder, E., Lookman, T., 2010. Thermodynamic theory of dislocation-mediated plasticity. Acta Mater. 58, 3718-3732. doi:10.1016/j.actamat.2010.03.009

Ledbetter, H.M., 1973. Estimation of Debye temperatures by averaging elastic coefficients. J. Appl. Phys. 44, 1451-1454. doi:10.1063/1.1662392

35 | P a g e 
Li, P., Li, S.X., Wang, Z.G., Zhang, Z.F., 2011. Fundamental factors on formation mechanism of dislocation arrangements in cyclically deformed fcc single crystals. Prog. Mater. Sci. 56, 328-377. doi:10.1016/j.pmatsci.2010.12.001

Li, S., Sauer, R.A., Wang, G., 2006a. The Eshelby Tensors in a Finite Spherical Domain-Part I: Theoretical Formulations. J. Appl. Mech. 74, 770-783. doi:10.1115/1.2711227

Li, S., Wang, G., Sauer, R.A., 2006b. The Eshelby Tensors in a Finite Spherical Domain-Part II: Applications to Homogenization. J. Appl. Mech. 74, 784-797. doi:10.1115/1.2711228

Lloyd, J.T., 2010. Implications of limited slip in crystal plasticity (Thesis). Georgia Institute of Technology.

Lukas, P., Kunz, L., Svoboda, M., 1999. Stress-strain response and fatigue life of copper single crystals cyclically loaded with a positive mean stress. Mater. Sci. Eng. A 272, 31-37.

Mecke, K., Blochwitz, C., 1982. Saturation Dislocation Structures in Cyclically Deformed Nickel Single Crystals of Different Orientations. Cryst. Res. Technol. 17, 743-758. doi:10.1002/crat.2170170610

Mitchell, T.E., 1964. Dislocations and Plasticity in Single Crystals of Face-Centered Cubic Metals and Alloys. Prog. Appl. Mater. Res. 6, 117-237.

Morrison, D.J., 1994. Influence of grain size and texture on the cyclic stress-strain response of nickel. Mater. Sci. Eng. A 187, 11-21. doi:10.1016/0921-5093(94)90326-3

Morrison, D.J., Aubin, V., 1994. Cyclic stress-strain response of polycrystalline nickel. Mater. Sci. Eng. A 177, 29-42. doi:doi: DOI: 10.1016/0921-5093(94)90475-8

Morrison, D.J., Moosbrugger, J.C., 1997. Effects of grain size on cyclic plasticity and fatigue crack initiation in nickel. Int. J. Fatigue 19, 51-59.

Mughrabi, H., 1987. The long-range internal stress field in the dislocation wall structure of persistent slip bands. Phys. Status Solidi A 104, 107-120. doi:10.1002/pssa.2211040108

Mughrabi, H., 1979. Microscopic mechanisms of metal fatigue, in: Haasen, P., Gerold, V., Kostorz, G. (Eds.), The Strength of Metals and Alloys. Pergamon Press, pp. 1615-39.

Mughrabi, H., Hoppel, H.W., 2010. Cyclic deformation and fatigue properties of very fine-grained metals and alloys. Int. J. Fatigue, Emerging Frontiers in Fatigue 32, 1413-1427. doi:10.1016/j.ijfatigue.2009.10.007

Mughrabi, H., Pschenitzka, F., 2005. Constrained glide and interaction of bowed-out screw dislocations in confined channels. Philos. Mag. 85, 3029-3045. doi:10.1080/14786430500079975

Mura, T., 1987. Micromechanics of defects in solids. Martinus Nijhoff Publishers, Dordrecht.

Nahm, H., Moteff, J., Diercks, D.R., 1977. Substructural development during low cycle fatigue of AISI 304 stainless steel at $649^{\circ} \mathrm{C}$. Acta Metall. 25, 107-116. doi:10.1016/0001-6160(77)90114-6

Narayanan, S., McDowell, D.L., Zhu, T., 2014. Crystal Plasticity Model for BCC Iron Atomistically Informed by Kinetics of Correlated Kinkpair Nucleation on Screw Dislocation. J. Mech. Phys. Solids 65, 5468. doi:10.1016/j.jmps.2014.01.004

Ohno, N., Wang, J.-D., 1993. Kinematic hardening rules with critical state of dynamic recovery, part I: formulation and basic features for ratchetting behavior. Int. J. Plast. 9, 375-390. doi:10.1016/0749-6419(93)90042-0

Ortiz, M., Repetto, E.A., 1999. Nonconvex energy minimization and dislocation structures in ductile single crystals. J. Mech. Phys. Solids 47, 397-462.

Oudriss, A., Feaugas, X., 2016. Length scales and scaling laws for dislocation cells developed during monotonic deformation of (001) nickel single crystal. Int. J. Plast. 78, 187-202. doi:10.1016/j.ijplas.2015.11.003

Patra, A., McDowell, D.L., 2012. Crystal plasticity-based constitutive modelling of irradiated bcc structures. Philos. Mag. 92, 861-887. doi:10.1080/14786435.2011.634855

36 | P a g e 
Pham, M.-S., Holdsworth, S.R., 2014. Evolution of Relationships Between Dislocation Microstructures and Internal Stresses of AISI $316 \mathrm{~L}$ During Cyclic Loading at $293 \mathrm{~K}$ and $573 \mathrm{~K}\left(20^{\circ} \mathrm{C}\right.$ and $\left.300{ }^{\circ} \mathrm{C}\right)$. Metall. Mater. Trans. A 45, 738-751. doi:10.1007/s11661-013-1981-7

Pham, M.S., Holdsworth, S.R., Janssens, K.G.F., Mazza, E., 2013. Cyclic deformation response of AISI 316L at room temperature: Mechanical behaviour, microstructural evolution, physically-based evolutionary constitutive modelling. Int. J. Plast. 47, 143-164. doi:10.1016/j.ijplas.2013.01.017

Polak, J., Helesic, J., Obrtlik, K., 1988. Nucleation stress for persistent slip bands in fatigued copper single crystals. Mater. Sci. Eng. A 101, 7-12.

Rao, S., Parthasarathy, T.A., Woodward, C., 1999. Atomistic simulation of cross-slip processes in model fcc structures. Philos. Mag. A 79, 1167-1192. doi:10.1080/01418619908210354

Rasmussen, T., Vegge, T., Leffers, T., Pedersen, O.B., Jacobsen, K.W., 2000. Simulation of structure and annihilation of screw dislocation dipoles. Philos. Mag. A 80, 1273-1290.

Ray, K.K., Mallik, A.K., 1983. An investigation on the activation volume for plastic flow in nickel determined by micro and macro tests. Phys. Status Solidi A 75, 451-458. doi:10.1002/pssa.2210750214

Sauzay, M., 2008. Analytical modelling of intragranular backstresses due to deformation induced dislocation microstructures. Int. J. Plast. 24, 727-745. doi:doi: DOI: 10.1016/j.ijplas.2007.07.004

Sauzay, M., 2007. Cubic elasticity and stress distribution at the free surface of polycrystals. Acta Mater. 55, 1193-1202. doi:doi: DOI: 10.1016/j.actamat.2006.09.035

Sauzay, M., Kubin, L.P., 2011. Scaling laws for dislocation microstructures in monotonic and cyclic deformation of fcc metals. Prog. Mater. Sci. 56, 725-784. doi:10.1016/j.pmatsci.2011.01.006

Sauzay, M., Liu, J., Rachdi, F., Signor, L., Ghidossi, T., Villechaise, P., 2014. Physically-Based Simulations of the Cyclic Behavior of FCC Polycrystals. Adv. Mater. Res. 891-892, 833-839. doi:10.4028/www.scientific.net/AMR.891-892.833

Schwartz, J., Fandeur, O., Rey, C., 2013. Numerical approach of cyclic behaviour of 316LN stainless steel based on a polycrystal modelling including strain gradients. Int. J. Fatigue 55, 202-212. doi:10.1016/j.ijfatigue.2013.07.003

Steckmeyer, A., Sauzay, M., Weidner, A., Hieckmann, E., 2012. Micromechanical modelling of the cyclic stress-strain behaviour of nickel polycrystals. Int. J. Fatigue 40, 154-167. doi:10.1016/j.ijfatigue.2011.10.019

Tabata, T., Fujita, H., Hiraoka, M.-A., Miyake, S., 1982. The relationship between flow stress and dislocation behaviour in [111] aluminium single crystals. Philos. Mag. A 46, 801-816. doi:10.1080/01418618208236932

Thiele, E., Klemm, R., Hollang, L., Holste, C., Schell, N., Natter, H., Hempelmann, R., 2005. An approach to cyclic plasticity and deformation-induced structure changes of electrodeposited nickel. Mater. Sci. Eng. A 390, 42-51. doi:10.1016/j.msea.2004.09.022

Tinga, T., Brekelmans, W.A.M., Geers, M.G.D., 2010. Cube slip and non-Schmid effects in single crystal Ni-base superalloys. Model. Simul. Mater. Sci. Eng. 18, 015005.

Tippelt, B., Breitschneider, J., Hähner, P., 1997. The Dislocation Microstructure of Cyclically Deformed Nickel Single Crystals at Different Temperatures. Phys. Status Solidi A 163, 11-26. doi:10.1002/1521-396X(199709)163:1<11::AID-PSSA11>3.0.CO;2-X

Toth, L.S., Estrin, Y., Molinari, A., 2002. Strain Hardening at Large Strains as Predicted by Dislocation Based Polycrystal Plasticity Model. J. Eng. Mater. Technol. 124, 71-77. doi:10.1115/1.1421350

Verdier, M., Fivel, M., Groma, I., 1998. Mesoscopic scale simulation of dislocation dynamics in fcc metals: Principles and applications. Model. Simul. Mater. Sci. Eng. 6, 755. doi:10.1088/09650393/6/6/007 
Wang, Zhirui, Gong, B., Wang, Zhongguang, 1997. Cyclic deformation behavior and dislocation structures of [001] copper single crystals-II. Characteristics of dislocation structures. Acta Mater. 45, 1379-1391. doi:10.1016/S1359-6454(96)00289-3

Wang, Z.Q., Beyerlein, I.J., LeSar, R., 2007. The importance of cross-slip in high-rate deformation. Model. Simul. Mater. Sci. Eng. 15, 675. doi:10.1088/0965-0393/15/6/006

Wen, W., Borodachenkova, M., Tome, C.N., Vincze, G., Rauch, E.F., Barlat, F., Grácio, J.J., 2016. Mechanical behavior of low carbon steel subjected to strain path changes: Experiments and modeling. Acta Mater. 111, 305-314. doi:10.1016/j.actamat.2016.03.075

Yagodzinskyy, Y., Saukkonen, T., Hanninen, H., 2008. Effect of hydrogen on plastic strain localization in single crystals of nickel and austenitic stainless steel, in: Somerday, B.P., Sofronis, P., Jones, R.C. (Eds.), . Presented at the International Hydrogen Conference, Grand Teton National Park, Wyoming, USA, pp. 97-104.

Yuan, C., Guo, J.T., Yang, H.C., Wang, S.H., 1998. Deformation mechanism for high temperature creep of a directionally solidified nickel-base superalloy. Scr. Mater. 39, 991-997. doi:10.1016/S13596462(98)00255-3

Zaiser, M., Sandfeld, S., 2014. Scaling properties of dislocation simulations in the similitude regime. Model. Simul. Mater. Sci. Eng. 22, 065012. doi:10.1088/0965-0393/22/6/065012

Zhou, C., Biner, S.B., LeSar, R., 2010. Discrete dislocation dynamics simulations of plasticity at small scales. Acta Mater. 58, 1565-1577. doi:10.1016/j.actamat.2009.11.001 
2017-06-21

\section{Mesoscale cyclic crystal plasticity with dislocation substructures}

Castelluccio, Gustavo M.

Elsevier

Gustavo M. Castelluccio, David L. McDowell, Mesoscale cyclic crystal plasticity with dislocation substructures, International Journal of Plasticity, Vol. 98, November 2017, pp. 1-26

http://dx.doi.org/10.1016/j.ijplas.2017.06.002

Downloaded from Cranfield Library Services E-Repository 